\title{
Equilibria in systems of social interactions
}

\author{
Ulrich Horst $^{\mathrm{a}}$, José A. Scheinkman ${ }^{\mathrm{b}, \mathrm{c}, *}$

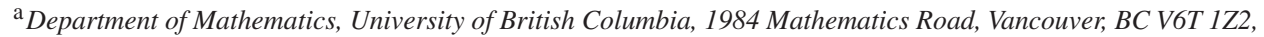 \\ Canada \\ ${ }^{\mathrm{b}}$ Department of Economics, Princeton University, 26 Prospect Avenue, Princeton, NJ 08540-5296, USA \\ ${ }^{\mathrm{c}}$ National Bureau of Economic Research, 1050 Massachusetts Ave., Cambridge, MA 02138, USA
}

Received 20 February 2003; final version received 25 February 2005

Available online 1 June 2005

\begin{abstract}
In this paper, we establish existence and uniqueness results for equilibria in systems with an infinite number of agents and with local and global social interactions. We also examine the structure of the equilibrium distribution and derive a "Markov"property for the equilibrium distribution of a class of spatially homogeneous systems.
\end{abstract}

(C) 2005 Elsevier Inc. All rights reserved.

JEL classification: C62; D50; D71

Keywords: Existence of equilibria; Local interaction; Random interaction structure; Social interactions

\section{Introduction and overview}

A common problem in the social sciences is the observation of large differences in outcomes in the absence of corresponding differences in fundamentals. To accommodate such phenomena a model must necessarily produce a big "multiplier" that transforms small changes in exogenous variables into large changes in endogenous variables. Models of social interactions are capable of displaying large multipliers. In these models an agent's behavior depends, among other things, on choices of other agents in a reference group and/or on the expectations that the agent has of the behavior of other agents. If the marginal utility of undertaking an action increases as other agents undertake more of the same action,

\footnotetext{
* Corresponding author.

E-mail addresses: horst@math.ubc.ca (U. Horst), joses@ princeton.edu (J.A. Scheinkman).
} 
a change in fundamentals has a direct effect on behavior and an indirect effect that are of the same sign. If the indirect effect is significant enough, we obtain a large multiplier that may explain radically different outcomes from slightly distinct fundamentals.

This property of models of social interactions has resulted in a large literature that uses these models to explain economic and social behavior. Examples include the prevalence of segregation [31,32], the adoption of religions [2], business cycles [6], economic growth [7], the variance of crime rates across space [17], technology adoption [9] or price fluctuations in financial markets $[15,22]$.

These papers and much of the literature on social interactions assume very special interaction structures. However, [18] contains existence and uniqueness of equilibria results for a class of models of social interactions with a finite number of agents with fairly general interaction structure. Existence and uniqueness results have also been established for "large" systems of social interactions where agents care only about some weighted average behavior of all other agents; see, e.g., [4,5,18] or [27]. Interaction games with an infinite number of players in which each player interacts with a finite number of neighbors, but interactions are otherwise general, are discussed in [29,30]. Nonetheless there is a scarcity of results concerning "large" systems with arbitrary local and global interactions.

In many examples in the literature agents care about the average behavior throughout the population. In these cases, the analysis is more naturally done in the context of an infinity of agents. ${ }^{1}$ In other examples an agent's utility is affected by the choices of some particular nearby agents. In this paper we consider systems with general interaction structures. Agents interact locally with a set of other agents, their "neighbors," and globally with the whole population. The local interactions are expressed by the fact that an agent's utility is affected by the choices of his neighbors. An agent's utility also depends on the empirical distribution of actions across the entire population, reflecting global interactions. This distinction is unnecessary for finite systems, but plays an important role in the analysis of systems with an infinite number of agents.

The systems of social interactions in this paper have random interaction structures and random taste distributions. Individuals choose actions after the realizations of these random variables. As in [17] or [25] an agent's utility is a function of the actual actions chosen by their neighbors, but here utility can also be affected by the distribution of actions throughout the population.

For systems with an infinite number of agents, the question of existence of equilibrium involves two sets of issues. The continuity of an agent's utility function with respect to the vector of actions by all other agents in the appropriate topology requires implicitly, as we argue in Section 2, that the dependence of his utility function on another agent's action decays sufficiently fast as the distance to that other agent grows. When all interactions are local the continuity of the utility functions together with the standard convexity assumptions suffice. For infinite systems in which global interactions are present the analysis is more delicate. In fact, a good portion of this paper is dedicated to establishing existence and uniqueness results for a class of infinite systems with both local and global interactions. If some form of spatial homogeneity prevails, we establish sufficient conditions that insure the

\footnotetext{
${ }^{1}$ Systems with an infinity of agents can also be seen as limits of large finite systems. For formal results establishing this connection see [23].
} 
existence or uniqueness of equilibria. These conditions restrict the influence of an agent's choices on the optimal decisions of other agents, and are variations on the moderate social influence (MSI) assumption that was used by Glaeser and Scheinkman in [18] to establish uniqueness of equilibrium for finite systems. For some special cases, we translate these requirements into assumptions on the utility functions. A short description of our approach to the existence and uniqueness question is given at the beginning of Section 4.1.

We also study the structure of the equilibrium distribution and show that if the interactions are only local and with a maximum radius $M$, and all shocks are independent and identically distributed, then the law of the equilibrium profile is Markov of order $2 M$, that is knowledge of the actions taken by agents that are further than $2 M$ away does not help predict the action of an agent if one already knows the actions of all agents that are at most $2 M$ away. A simple example shows that the law of the equilibrium profile is not, in general, Markov of order $M$. This example shows that the distributions of equilibrium action profiles is not specified by the family of individual best response functions. Observations of the choices of agents that are not neighbors of $a$ can help predict the choice of action by $a$. For systems with both local and global interactions one cannot hope for a Markov property. However a Markov property holds conditional on the empirical distribution of actions.

The rest of the paper proceeds as follows. In Section 2 we define formally the systems of social interactions that we will consider. Section 3 shows that our framework includes many well known examples in the literature. Section 4 contains the main results. The structure of the equilibria is studied in Section 5. All proofs appear in appendices.

\section{Systems of random social interactions}

In this section, we define systems of random social interactions. Each agent is indexed by an $a \in \mathbb{A}$, where $\mathbb{A}$ is a subset of the lattice $\mathbb{Z}^{d}$ of $d$-dimensional vectors with integer entries. An agent will choose an action $x^{a}$ from a common compact and convex set of possible actions $X$. An action profile $x \in S:=\left\{x=\left\{x^{b}\right\}_{b \in \mathbb{A}}: x^{b} \in X\right\}$ is a list of actions $x^{b}$ for each $b \in \mathbb{A}$. The configuration space $S$ of all action profiles is equipped with the product topology, and hence it is compact. In our model the utility function $U^{a}$ of the agent $a \in \mathbb{A}$ may also depend on the actions chosen by other agents $b \in \mathbb{A}$. In addition, the utility function of each agent is random, that is, it also depends on the realization of a random variable $\vartheta^{a}$ defined on a probability space $(\Omega, \mathcal{F}, \mathbb{P})$. In short, agent $a$ 's utility is given by $U^{a}\left(x^{a},\left\{x^{b}\right\}_{b \neq a}, \vartheta^{a}\right)$.

In models of social interactions, the influence of other agents' actions appear in at least two distinct ways. The first is the impact of the actions of a particular set of "neighbors." Another is through a statistic, often the average action, of the distribution of actions of all agents. We call the former the local component of social interactions and the latter the global component. To be able to describe these distinct influences, and to allow for variable degrees of influence on the utility of an agent by the choices of his neighbors, we write $\vartheta^{a}=\left(J^{a}, \theta^{a}\right)$, for each agent $a \in \mathbb{A}$. The random variable $\theta^{a}$ describes a taste shock and assumes values in a set $\Theta$. The random variable $J^{a}=\left(J^{a, b}\right)_{b \neq a}$, takes values in the set $\Xi:=\mathbb{R}^{\mathbb{A} \backslash\{0\}}$. The realization of the random variable $J^{a, b}$ defines the effect the choice of the neighbor $b \neq a$ has on the utility of the agent $a \in \mathbb{A}$. To accommodate the global 
component, the utility function also depends on the empirical distribution $\varrho(x)$ associated with the action profile $x$. More precisely the utility function of each agent is a (measurable) mapping

$$
\left(x, J^{a}, \theta^{a}\right) \mapsto U^{a}\left(x^{a},\left\{x^{b}\right\}_{b \neq a}, J^{a}, \theta^{a}\right) \equiv u^{a}\left(x^{a},\left\{J^{a, b} x^{b}\right\}_{b \neq a}, \varrho(x), \theta^{a}\right) .
$$

The representation of the utility function given by Eq. (1) is not necessarily unique, and at this point it is only useful as an interpretation of the interaction patterns of a system. We will see later, that for infinite systems, regularity (continuity) will require that the dependency of an agent's utility on other agents' actions decays fast enough as a function of the distance, and so it will place some restrictions on the random variables $J^{a}$. In addition, in the infinite case, not all profiles have an associated empirical distribution. We will deal with this problem below.

We call

$$
N(a):=\left\{b \in \mathbb{A}: J^{a, b} \neq 0\right\}
$$

the reference group, or the set of neighbors of the agent $a$. If $b \notin N(a)$ then the action of agent $b$ influences agent $a$ choices only through its impact on the distribution $\varrho(x)$. Different realizations of the random variables $J^{a}$ may yield different peer groups, and so different interaction patterns may emerge. This makes our approach considerably more general than the usual "nearest neighbor" and "mean field" interaction setups studied in e.g. [5,15,24,27].

The following example illustrate the concept of a random peer group.

Example 1. To each agent $a \in \mathbb{A}$ associate the "a-priori neighborhood"

$$
\tilde{N}(a):=\{b \in \mathbb{A}:|a-b| \leqslant M\} \cap \mathbb{A} \quad(l \in \mathbb{N}),
$$

i.e., a set of "candidates" that the agent $a$ may interact with. The actual peer group $N(a)$ of agent $a$ is the random subset of $\tilde{N}(a)$ defined by

$$
N(a):=\left\{b \in \mathbb{A}: J^{a, b}=1\right\},
$$

where $\left(J^{a, b}\right)_{b \in \tilde{N}(a)}$ are independent random variables taking the values " 1 " and " 0 " with probability $p$ and $1-p$, respectively.

The general definition of a utility function given in Eq. (1) is not very convenient for establishing the existence of an equilibrium in systems of social interactions. This is a consequence of the fact that a proof of existence of an equilibrium typically requires continuity of the utility functions and compactness of the configuration space $S$. In many interesting examples, the configuration $x \in S$ enters the utility function of an agent $a \in \mathbb{A}$ both through the actions $x^{b}$ taken by a finite set of neighbors $b \in N(a)$ and via the distribution of behavior throughout the entire population. In case of an infinite number of agents, $S$ is compact in the product topology, but if the utility function $u^{a}$ in Eq. (1) depends on $x$ through the empirical distribution $\varrho(x)$ in a non-trivial manner, it is not a continuous function of $x .^{2}$ In addition, a

\footnotetext{
${ }^{2}$ Since the class of continuous functions $f: S \rightarrow \mathbb{R}$ which depend only on finitely many coordinates is dense in the space $\mathcal{C}(S)$ of all continuous functions on $S$ equipped with the topology of uniform convergence, a map $f \in \mathcal{C}(S)$ depends on an action profile $x \in S$, at least approximately, only through finitely many actions $x^{b}$. In particular, the dependence on $x^{b}$ has to decay fast enough as $b \rightarrow \infty$.
} 
configuration $x$ does not necessarily have an empirical distribution. To solve these problems we apply a procedure introduced in Föllmer and Horst [14] and Horst [21] and previously applied in [22] to study stock price fluctuations in a microstructure model for financial markets. We separate the local and the global impact of an action profile $x=\left\{x^{a}\right\}_{a \in \mathbb{A}} \in S$ on an agent's utility and view the empirical distribution $\varrho(x)$ of individual actions associated with $x \in S$ as a parameter of the utility function. In this way we view the utility function $u^{a}$ as depending on an additional argument.

In order to make this more precise, we denote by $\mathcal{M}(X)$ the class of all probability measures on $X$. The space $\mathcal{M}(X)$ is compact with respect to the topology of weak convergence. ${ }^{3}$ In a system of social interactions, the utility function of an the agent $a \in \mathbb{A}$ is defined as a map $U^{a}: S \times \mathcal{M}(X) \times \Xi \times \Theta \rightarrow \mathbb{R}$, and required to be continuous in the product topology. This utility function will be assumed to be of the form given by (1) above, that is:

$$
U^{a}\left(x^{a},\left\{x^{b}\right\}_{b \neq a}, \varrho, J^{a}, \theta^{a}\right) \equiv u^{a}\left(x^{a},\left\{J^{a, b} x^{b}\right\}_{b \neq a}, \varrho, \theta^{a}\right) .
$$

Notice that, in principle, there is no consistency requirement between the $x \in S$ and the "empirical distribution" $\varrho$ on $X$, and in particular it is not necessary that we can associate an empirical distribution to the profile $x$. In equilibrium, we will require consistency.

We simplify our notation fixing the probability space $(\Omega, \mathcal{F}, \mathbb{P})$, making the canonical choice $(\Omega, \mathcal{F}, \mathbb{P})=\left((\Xi \times \Theta)^{\mathbb{A}}, \mathcal{B}(\Xi \times \Theta){ }^{\mathbb{A}}, \mathbb{P}\right)$ where $\mathcal{B}(\Xi \times \Theta)$ denotes the Borel $\sigma$-field on $\Xi \times \Theta$. Once the set of agents $\mathbb{A}$ is fixed, all variations are coded in the probability distribution $\mathbb{P}$, and we are now ready to introduce our definition of systems of random social interactions.

Definition 2. A system of random social interactions is a vector

$$
\mathcal{E}=\left(\mathbb{A}, \mathbb{P}, X,\left(U^{a}\right)_{a \in \mathbb{A}}\right)
$$

with the following components:

(i) $\mathbb{A} \subset \mathbb{Z}^{d}$ is a set of agents.

(ii) $\mathbb{P}$ is a probability measure on $(\Omega, \mathcal{F})$.

(iii) $X \subset \mathbb{R}^{l}$ is a common compact convex action space.

(iv) $U^{a}: S \times \mathcal{M}(X) \times \Xi \times \Theta \rightarrow \mathbb{R}$ is a measurable mapping such that (2) holds and such that for $\mathbb{P}$-a.e. pair $(J, \theta)$, the map

$$
(x, \varrho) \mapsto U^{a}\left(x^{a},\left\{x^{b}\right\}_{b \neq a}, \varrho, J^{a}, \theta^{a}\right)
$$

is continuous and concave in $x^{a}$; the utility function of agent $a \in \mathbb{A}$.

Our notion of a random system of social interactions includes systems with finitely many agents. There are also cases in which the utility of the agents can be defined in a continuous

${ }^{3}$ A sequence $\left\{\mu_{t}\right\}_{t \in \mathbb{N}}$ of probability measures converges weakly to $\mu \in \mathcal{M}(X)$ if and only if

$$
\lim _{t \rightarrow \infty} \int_{X} f(x) \mu_{t}(d x)=\int_{X} f(x) \mu(d x)
$$

for all continuous functions $f: X \rightarrow \mathbb{R}$. 
manner without dependence on the argument $\varrho$, the distribution of actions. If the system has a representation satisfying all conditions of Definition 2, in which the utility functions do not depend on $\varrho$, we will say that the system is purely local.

Moreover $U^{a}\left(\cdot, \varrho, J^{a}, \theta^{a}\right)$ is continuous on $S$ if and only if an agent's utility depends continuously on the actions $\left\{x^{b}\right\}_{b \neq a}$ taken by all the other agents and if, in addition, the dependence of $U^{a}$ on the action $x^{b}$ taken by the agent $b$ decays sufficiently fast. ${ }^{4}$ In this sense continuity of the utility function translates into an assumption on the interaction patterns. This justifies our earlier claim that what we call the local component of interactions has to decay fast enough. 5

It should be emphasized that for equilibrium analysis it is equivalent to use a utility function that depends only on the action profile $x$, or to use the "extended" continuous utility function that involves the variables $(x, \varrho)$ since, in equilibrium, we will require that $\varrho$ equals the empirical distribution of the action profile $x$.

\section{Examples}

In this section we show how our definition of a homogeneous random systems of social interactions fits into the frameworks analyzed in $[19,4,13]$, respectively. To this end, we denote by

$$
m(\varrho):=\int_{X} x \varrho(x)
$$

the expected action associated to the measure $\varrho$. In an infinite system, $m(\varrho)$ turns out to be the average action associated to an equilibrium action profile.

\subsection{Mean-field systems}

In mean field systems the utility an agent enjoys depends on the actions taken by other agents only through the empirical distribution of all actions-all interactions are global. In our setup it is natural to use the following definition:

Definition 3. $\mathcal{E}$ is a mean-field system if for each $a \in \mathbb{A}$

$$
U^{a}\left(x^{a},\left\{x^{b}\right\}_{b \neq a}, \varrho, J^{a}, \theta^{a}\right)=U^{a}\left(x^{a}, \varrho, \theta^{a}\right) .
$$

Example 4. Continuous action versions of the models studied in $[4,8]$ may be viewed as mean-field systems in the sense of Definition 3. These papers treat systems with finitely many agents and assume that an agents' utility function is, in our notation, of the form

$$
U^{a}\left(x^{a}, \varrho, \theta^{a}\right)=u\left(x^{a}\right)+\frac{1}{|\mathbb{A}|} \sum_{b \in \mathbb{A}} x^{a} \mathbb{E}_{a} x^{b}+f\left(x^{a}, \theta^{a}\right),
$$

\footnotetext{
${ }^{4}$ See Footnote 2 above.

5 The results in [29] require the stronger assumption that each agent interacts only with a finite number of neighbors.
} 
where $\mathbb{E}_{a} x^{b}$ denotes the expectation of agent $a$ about the behavior of agent $b \in \mathbb{A}$. In particular, an agents' utility does not depend on the actions taken by his neighbors, but only on his expectation about their behavior. Brock and Durlauf argue in [4] that in their spatially homogeneous setup, in equilibrium, all agents share the same expectations about the behavior of their neighbors. This means that, in equilibrium, $\mathbb{E}_{a} x^{b}=m$ for some $m \in X$ and for all $a, b \in \mathbb{A}$. Thus, for $|\mathbb{A}| \rightarrow \infty$, the system with the alternative utility function

$$
U^{a}\left(x^{a}, \varrho, \theta^{a}\right)=u\left(x^{a}\right)+x^{a} m(\varrho)+f\left(x^{a}, \theta^{a}\right)
$$

has equilibria that contain all equilibria of the original system. Hence, for $|\mathbb{A}| \rightarrow \infty$ any property that is true for equilibria of systems of random social interactions with utilities of the form given in Eq. (5), holds automatically for systems with utility functions described by Eq. (4).

\subsection{Föllmer's model of endogenous preference formation}

Föllmer's pioneering examination of Ising economies [13] with endogenous preferences can be fit into our description of a mean-field system. Let $\Theta=\{-1,1\}$, assume agents can choose an action $x \in X=[-1,1]$, and that

$$
U^{a}\left(x, \varrho, \theta^{a}\right)=x^{a} \theta^{a} .
$$

The linear form of the utility function guarantees that each agent will always choose $x \in$ $\{-1,1\}$, and as in [13] we may interpret the taste shock as defining the preference for one of two possible consumption goods. Agents are indexed by the two-dimensional integer lattice and interact with their nearest neighbor. That is $J^{a, b}=1$ for $\max _{k=1,2}\left|a^{k}-b^{k}\right|=1$, and $J^{a, b}=0$ otherwise. The preferences of an agent $a \in \mathbb{A}$ depend in a random manner on the preferences of his neighbors. More precisely, fix a local specification, i.e., a family of stochastic kernels $\left(\pi^{a}\right)_{a \in \mathbb{A}}$ from $\Theta^{4}$ to $\Theta$. These stochastic kernels describe the conditional probability of an agent's taste shock $\theta^{a}$ conditional on the taste shocks of his four neighbors, that is $\pi^{a}=\pi^{a}\left(\cdot ;\left\{\theta^{b}\right\}_{b \in N(a)}\right)$. Föllmer calls a measure $\mu$ on the configuration space $\Theta^{\mathbb{A}}$ a global phase if $\mu$ is consistent with the local specification $\left(\pi^{a}\right)_{a \in \mathbb{A}}$ in the sense that

$$
\mu\left(\cdot \mid\left\{\theta^{b}\right\}_{b \in N(a)}\right)=\pi^{a}\left(\cdot ;\left\{\theta^{b}\right\}_{b \in N(a)} \quad \mu\right. \text {-a.s. }
$$

and assumes the random variable $\theta$ is distributed according to some global phase $\mu$. There is an interactive structure in the distribution of the random preferences, but the utility of an agent does not depend on the actions taken by his neighbors.

\subsection{Models with local and global interaction}

The next example exhibits both local and global interaction.

Example 5. This is a generalization of the model studied in [19]. Here $X=[-1,1]$ and the interaction pattern is as in Example 1 above. Preference shocks are described by independent and identically distributed random variables $\theta^{a}(a \in \mathbb{A}$ on $\mathbb{R})$. Fix positive constants $p$, 
$\beta_{1}$ and $\beta_{2}, \beta_{1}+\beta_{2}<1$, and let

$$
\begin{aligned}
U^{a}\left(x^{a},\left\{x^{b}\right\}_{b \neq a}, \varrho, J^{a}, \theta^{a}\right)= & -\frac{1-\beta_{1}-\beta_{2}}{2}\left(x^{a}\right)^{2}-\frac{\beta_{1}}{2}\left(x^{a}-m(\varrho)\right)^{2} \\
& -\frac{\beta_{2}}{2}\left(x^{a}-\frac{1}{M} \sum_{b \in N(a)} J^{a, b} x^{b}\right)^{2}-\left(\theta^{a}-p\right) x^{a} .
\end{aligned}
$$

In particular, an agent's utility depends on the actions $x^{b}$ taken by his neighbors $b \in N(a)$ only through a suitably weighted average action, and so the impact of an individual agents' action on the utility of his neighbor tends to zero as $M \rightarrow \infty$.

The following example shows how our model can accommodate the framework considered in $[8,24]$.

Example 6. Let $X=[-1,1]$ and $N(a)=\{b \neq a:|a-b| \leqslant M\}$. The random variables $J^{a, b} \in[-1,1]$ and $\theta^{a}(a \in \mathbb{A}, b \in N(a))$ are independent and identically distributed. Let $\beta>0$ and $B>0$, and

$$
U^{a}\left(x^{a},\left\{x^{b}\right\}_{b \neq a}, \varrho, J^{a}, \theta^{a}\right)=x^{a} \beta \sum_{b \in N(a)} J^{a, b} x^{b}+B x^{a} m(\varrho)+\frac{1}{2} x^{a} \theta^{a} .
$$

The random variables $J^{a, b}$ specify the effect of an increase in the action by a neighbor $b$ on the marginal utility of an agent's $a$ own action. In contrast to the utility function given by Eq. (4), in this example the utility of an agent depends on the actions taken by his neighbors and not on his expectation about his neighbors actions.

\section{Equilibria in systems with random social interactions}

In this section we state results on existence and uniqueness of equilibria in systems of random social interactions, present some examples and an informal discussion of social multipliers. As usual, an equilibrium will be defined as a random configuration of actions where no agent can increase his own utility by deviating from the proposed action profile. In addition, if an agent's utility function depends directly on the empirical distribution of actions, the empirical distribution of equilibrium actions must coincide (almost surely) with the empirical distribution used by all agents when choosing their optimal action.

Definition 7. A random variable $g(J, \theta)=\left\{g^{a}(J, \theta)\right\}_{a \in \mathbb{A}}$ is an equilibrium for $\mathcal{E}=$ $\left(\mathbb{A}, \mathbb{P}, X,\left(U^{a}\right)_{a \in \mathbb{A}}\right)$ if:

(i) When $\mathcal{E}$ is not purely local, the empirical distribution associated with the action profile $g(J, \theta)$ exists almost surely, i.e., the weak limit

$$
\lim _{n \rightarrow \infty} \frac{1}{\left|\mathbb{A}_{n}\right|} \sum_{a \in \mathbb{A}} \delta_{g^{a}(J, \theta)}(\cdot)=\varrho(J, \theta)
$$

exists almost surely for some random variable $\varrho(J, \theta) \in \mathcal{M}(X)$ along the increasing sequence of finite sets $\mathbb{A}_{n}:=[-n, n]^{d} \cap \mathbb{A} \uparrow \mathbb{A}$. 
(ii) No agent has an incentive to deviate from the proposed strategy. That is, almost surely

$$
g^{a}(J, \theta) \in \arg \max _{x^{a} \in X} U^{a}\left(x^{a},\left\{g^{b}(J, \theta)\right\}_{b \neq a}, \varrho(J, \theta), J^{a}, \theta^{a}\right) \quad(a \in \mathbb{A}) .
$$

The requirement that the empirical distribution associated with an equilibrium exists is not imposed on purely local systems. However, as we will see in Theorem 19 below, in the equilibrium of some purely local systems the empirical distribution of actions does exist.

\subsection{Existence of equilibria in random systems of social interactions}

In this section, we present existence and uniqueness results. All proofs are in Appendix A. If interactions are purely local, existence follows from the assumed continuity and concavity of the utility function, and the compactness and convexity of the action space $X$, via a standard fixed-point argument (see Proposition 13). Recall however that, in the case of an infinite number of agents, the continuity of the utility functions already imposes a decay rate on the strength of interactions. When agents interact both in a local and in a global manner, we have the extra requirement that, in equilibrium, the agents' forecast of the empirical distribution of choices must equal the actual distribution of actions. In this instance, the question of existence is more delicate and requires additional assumptions.

Unless some form of spatial homogeneity prevails, there is no reason to expect that the empirical distribution associated with the equilibrium actions exists. For this reason, when global interactions are present, we will consider only homogeneous systems, as in Definition 8 below. In addition, homogeneous systems can be written as "mixtures" of ergodic systems and as a consequence the analysis of equilibria of homogeneous systems can be reduced to that of ergodic systems (see Proposition 11).

We separate the existence questions in two parts. First we fix the global distribution of actions $\varrho$ that agents anticipate and look for a configuration of actions $x$ in which each agent $a$ is maximizing his utility at $x^{a}$ given the action of all other agents $\left\{x^{b}\right\}_{b \neq a}$, and the anticipated distribution of actions $\varrho$. We call such a configuration a microscopic equilibrium. In a microscopic equilibrium the empirical distribution of the actions $x^{a}$ may not even exist. A microscopic equilibrium with an anticipated global distribution $\varrho^{*}$ is an equilibrium if $\varrho^{*}$ is the empirical distribution of the actions $x^{a}$. Even for non-homogeneous systems, for any given $\varrho$, microscopic equilibria always exist under our assumptions and this establishes, in particular, the existence of an equilibrium for purely local systems (Proposition 13). However this microscopic equilibrium need not be spatially homogeneous even if the system of social interactions is ergodic, and hence the empirical distribution of equilibrium actions may fail to exist. Proposition 15 establishes that homogeneity follows if the microscopic equilibria is unique for a given anticipated empirical distribution $\varrho$. To establish this uniqueness we need to bound the strength of interactions. Once the uniqueness of microscopic equilibria is guaranteed, the same bounds on the strengths of interactions are used to show that the microscopic equilibria vary continuously with the anticipated distribution of actions $\varrho$, and again a fixed point argument is used to produce an equilibrium for the system of social interactions.

We use two related assumptions that limit the influence of both the actions of other agents and the anticipated empirical distribution of actions on the choices of an agent. The first, 
MSI (Definition 17), must hold for every realization of $(J, \theta)$. Moderate social interactions suffice for the existence of a unique, and hence homogeneous, microscopic equilibria for any given anticipated distribution $\varrho$ (Proposition 18). Theorem 19 establishes that for ergodic systems that satisfy the MSI condition, there exists a spatially homogeneous equilibrium whose associated empirical distribution is almost surely independent of the realization of the random shocks. Furthermore, a strengthening of the MSI assumption guarantees uniqueness of equilibria. The second set of bounds on the strength of interactions, average moderate social influence (AMSI) (Definition 22) must only hold in expected value. For systems with one-sided interactions (see Definition 20) AMSI suffices to establish existence of equilibrium (see Proposition 23 and Theorem 24). Again uniqueness follows form a stronger version of the AMSI assumption.

\subsubsection{Homogeneous random systems}

In a homogeneous random system the utilities that agents enjoy are invariant under the simultaneous shifts of agents, their private taste shocks and interaction profiles. In particular, homogeneity rules out the existence of a single agent that always serves as a leader for all others. To precise the notion of a homogeneous system, we recall that $(\Omega, \mathcal{F}, \mathbb{P})$ denotes the canonical probability space, and introduce the shift transformations $T^{a}$ defined by

$$
T^{a}(J, \theta)=T^{a}\left(\left\{J^{b}, \theta^{b}\right\}_{b \in \mathbb{A}}\right):=\left\{\left(J^{b-a, c-a}\right)_{c \in \mathbb{A}}, \theta^{b-a}\right\}_{b \in \mathbb{A}}=:\left(T^{a} J, T^{a} \theta\right) .
$$

Definition 8. $\mathcal{E}=\left(\mathbb{A}, \mathbb{P}, X,\left(U^{a}\right)_{a \in \mathbb{A}}\right)$ is homogeneous if $\mathbb{A}=\mathbb{Z}^{d}$ and:

(i) There exists a measurable mapping $U: S \times \mathcal{M}(X) \times \Xi \times \Theta \rightarrow \mathbb{R}$ such that for all $a \in \mathbb{A}$

$$
U^{a}\left(x^{a},\left\{x^{b}\right\}_{b \neq a}, \varrho, J^{a}, \theta^{a}\right)=U\left(x^{a},\left\{x^{b}\right\}_{b \neq a}, \varrho, T^{a} J, T^{a} \theta\right) .
$$

(ii) The distribution of the random variable $(J, \theta)=\left\{\left(J^{a}, \theta^{a}\right)\right\}_{a \in \mathbb{A}}$ is stationary, i.e.,

$$
\mathbb{P}[(J, \theta) \in B]=\mathbb{P}\left[T^{a}(J, \theta) \in B\right]
$$

for all $a \in \mathbb{A}$ and for each measurable set $B \in \mathcal{F}$.

For a homogeneous system it is natural to search for an equilibrium that also displays homogeneity, that is where the equilibrium actions are invariant under the simultaneous shift of agents, their private taste shocks and interaction profiles.

Definition 9. An action profile $\left\{x^{a}(J, \theta)\right\}_{a \in \mathbb{A}}$ is homogeneous if

$$
x^{0}\left(T^{a} J, T^{a} \theta\right)=x^{a}(J, \theta)
$$

for each $a \in \mathbb{Z}^{d}$. An equilibrium configuration $g(J, \theta)$ is homogeneous if $g(J, \theta)$ is an homogeneous action profile.

Besides its natural appeal in the case of homogeneous systems, it turns out that homogeneous action profiles guarantee the existence of empirical distributions that is required to test the equilibrium condition given by Eq. (9) in Definition 7. 


\subsubsection{Ergodic random systems}

Homogeneous system can be written as "convex-combinations" of systems with probability measures that are ergodic in the sense of the following definition.

Definition 10. The homogeneous system $\mathcal{E}$ is ergodic if, in addition, the probability measure $\mathbb{P}$ is ergodic, that is if $\mathbb{P}$ satisfies a 0 -1-law on the $\sigma$-field of all shift invariant events.

Ergodicity includes the case where $\left(J^{a}, \theta^{a}\right)(a \in \mathbb{A})$ are independent and identically distributed random variables.

Homogeneous systems of social interactions may be viewed as mixtures of ergodic systems. Given a homogeneous system $\mathcal{E}=\left(\mathbb{A}, \mathbb{P}, X,\left(U^{a}\right)_{a \in \mathbb{A}}\right)$, there exists a set $\mathcal{M}_{0}$ of ergodic probability measures on $(\Omega, \mathcal{F})$ and a mixing measure $\pi$ such that ${ }^{6}$

$$
\mathbb{P}(\cdot)=\int_{\mathcal{M}_{0}} v(\cdot) \pi(d v) .
$$

In addition the measures $v \in \mathcal{M}_{0}$ are mutually singular: There exists (almost surely) mutually disjoint sets $\Omega_{v}$ such that

$$
v\left(\Omega_{v}\right)=1 \text { and } v\left(\Omega_{\hat{v}}\right)=0 \text { for } v \neq \hat{v} .
$$

The system $\mathcal{E}$ can thus be viewed as a mixture of ergodic systems $\mathcal{E}_{v}=\left(\mathbb{A}, v, X,\left(U^{a}\right)_{a \in \mathbb{A}}\right)$, and we may think of a homogeneous system in two steps. First nature chooses an ergodic measure $v$ using the mixture measure $\pi$. Second it chooses an interaction pattern $J$ and a vector of taste shocks $\theta$ according to $v$.

The following result shows that the equilibrium analysis of homogeneous systems can be reduced to the analysis of ergodic systems. The proof appears in Appendix A.1.

Proposition 11. Let $\mathcal{E}$ be a homogeneous system of random social interactions with an associated "ergodic decomposition" $\left(\mathcal{E}_{v}\right)_{v \in \mathcal{M}_{0}}$.

(i) If $g$ is a homogeneous equilibrium for $\mathcal{E}$, then $g$ coincides almost surely with a homogeneous equilibrium $g_{v}$ for $\mathcal{E}_{v}$ on $\Omega_{v}$.

(ii) Iffor every $v, g_{v}$ is a homogeneous equilibrium for $\mathcal{E}_{v}$, then the random variable g given by

$$
g(J, \theta)=g_{v}(J, \theta) \quad \text { if } \quad(J, \theta) \in \Omega_{v}
$$

defines a homogeneous equilibrium for $\mathcal{E}$.

\subsubsection{Microscopic equilibria}

When global interactions are present, the existence of an equilibrium for an ergodic system can be conceptually divided in two parts. The first part consists of taking as given for each $(J, \theta)$ an empirical distribution of actions and asking whether some prescribed profile of actions maximizes utility of each agent when he takes as given the actions of

\footnotetext{
${ }^{6}$ See Theorems 14.5 and 14.10 in [16] for further details.
} 
his neighbors and the given empirical distribution. The second part consists in checking whether the actions assigned to each agent generate the prescribed empirical distribution.

To separate the two problems, we introduce the notion of a microscopic equilibrium for a system $\mathcal{E}$.

Definition 12. For $\varrho \in \mathcal{M}(X)$, an action profile $g(\varrho, J, \theta)=\left\{g^{a}(\varrho, J, \theta)\right\}_{a \in \mathbb{A}}$ is a microscopic equilibrium associated with $\varrho$ if

$$
g^{a}(\varrho, J, \theta) \in \arg \max _{x^{a} \in X} U^{a}\left(x^{a},\left\{g^{b}(\varrho, J, \theta)\right\}_{b \neq a}, \varrho, J^{a}, \theta^{a}\right) \quad \mathbb{P} \text {-a.s. }
$$

for all $a \in \mathbb{A}$.

Thus, a microscopic equilibrium associated with $\varrho \in \mathcal{M}(X)$ is an action profile where each agent maximizes his utility given the actions taken by all the other agents and given the common anticipated distribution $\varrho$ of actions throughout the entire system. What distinguishes a microscopic equilibrium $g(\varrho, J, \theta)$ from an equilibrium is the fact that the empirical distribution associated to the configuration $g(\varrho, J, \theta)$ does not necessarily coincide with $\varrho$, and may even fail to exist. Of course, a microscopic equilibrium associated to some $\varrho^{*}$ is an equilibrium in the sense of our Definition 7 if

$$
\lim _{n \rightarrow \infty} \frac{1}{\left|\mathbb{A}_{n}\right|} \sum_{a \in \mathbb{A}_{n}} \delta_{g^{a}\left(\varrho^{*}, J, \theta\right)}(\cdot)=\varrho^{*} \quad \mathbb{P} \text {-a.s. }
$$

Since $\varrho^{*}$ is independent of $(J, \theta)$ a microscopic equilibrium will not be an equilibrium in the sense of our Definition 7 unless the microscopic equilibrium has an empirical distribution that is independent of $(J, \theta)$. As we will show below this property holds for a homogeneous microscopic equilibrium of an ergodic system.

The existence of a microscopic equilibrium for general systems of random social interactions follows from standard arguments, but there is no guarantee that this equilibrium is homogenous even when the system is ergodic.

Proposition 13. Let $\mathcal{E}$ be a system of random social interactions (not necessarily homogeneous). For all $\varrho \in \mathcal{M}(X)$ the system has a microscopic equilibrium $g(\varrho, \cdot)$ with respect to $\varrho$. In particular every purely local system $\mathcal{E}$ has an equilibrium.

\subsubsection{Existence and uniqueness of homogeneous microscopic equilibria}

As we argued previously, it is natural to look for homogeneous equilibria in ergodic systems. To establish the existence of a homogeneous microscopic equilibrium in infinite systems we need to place qualitative bounds on the strengths of interactions between different agents and on the dependence of agents' optimal choices on the perceived empirical distribution of actions. We will in fact place bounds on the reactions of an agent's choice to changes on another agent's action and to the anticipated distribution of actions.

In order to simplify notation, we will assume from now on that an agent's utility function is strictly concave with respect to his own action. The case where utility functions are concave, but not necessarily strictly concave, is discussed in Section 4.1.7 below. 
Assumption 14. The utility function $U$ in (11) is strictly concave with respect to its first argument. In particular, an agents conditional best reply $h^{a}$ is uniquely determined:

$$
h^{a}\left(\left\{x^{b}\right\}_{b \neq a}, \varrho, J, \theta\right):=\arg \max _{x^{a} \in X} U^{a}\left(x^{a},\left\{x^{b}\right\}_{b \neq a}, \varrho, J^{a}, \theta^{a}\right) .
$$

Strict concavity of the agents' utility functions with respect to their own actions along with homogeneity of the $U^{a}$ yields homogeneity of the best reply functions:

$$
h^{a}\left(\left\{x^{b}\right\}_{b \neq a}, \varrho, J, \theta\right)=h^{0}\left(\left\{x^{b-a}\right\}_{b \neq a}, \varrho, T^{a} J, T^{a} \theta\right) \quad(a \in \mathbb{A}) .
$$

This will allow us to state our weak interaction condition in terms of the best reply functions of the "reference agent" 0 .

The following lemma gives a simple sufficient condition for the existence of a homogeneous microscopic equilibrium, that is a microscopic equilibrium with a homogeneous allocation.

Lemma 15. If an ergodic system $\mathcal{E}$ has a unique microscopic equilibrium $g(\varrho, \cdot)$ associated with a distribution $\varrho$, then $g(\varrho, \cdot)$ is a homogeneous allocation.

Before we introduce the weak interaction conditions that guarantee existence of equilibria in infinite systems, we recall that the Vasserstein distance

$$
d_{V}(\varrho, \tilde{\varrho})=\sup \left\{\frac{\left|\int f d \varrho-\int f d \tilde{\varrho}\right|}{L(f)} \mid f: S \rightarrow R \text { Lipschitz with constant } L(f)\right\}
$$

induces the weak topology on $\mathcal{M}(X)$, and that $\left(\mathcal{M}(X), d_{V}\right)$ is a compact metric space. Here $L(f)$ denotes the Lipschitz constant of $f$ with respect to a metric that induces the product topology on the configuration space $S$. The next definition allows us to measure the dependence of an agent's best reply on another agent's action and the anticipated distribution of actions.

Definition 16. The best reply function $h^{0}$ is Lipschitz continuous if there are random variables $\left(L^{a}\right)_{a \in \mathbb{A}}$ whose sum is uniformly bounded, and a random variable $L^{\varrho}$ such that $\mathbb{P}$-a.s.

$$
\begin{aligned}
& \left|h^{0}\left(\left\{x^{a}\right\}_{a \neq 0}, \hat{\varrho}, J, \theta\right)-h^{0}\left(\left\{y^{a}\right\}_{a \neq 0}, \tilde{\varrho}, J, \theta\right)\right| \\
& \quad \leqslant \sum_{a \neq 0} L^{a}\left(J^{0}, \theta^{0}\right)\left|x^{a}-y^{a}\right|+L^{\varrho}\left(\theta^{0}\right) d_{V}(\hat{\varrho}, \tilde{\varrho}) .
\end{aligned}
$$

Given an interaction profile $J$ and a configuration of taste shocks $\theta$, the quantity $L^{a}$ may be viewed as a bound for the influence that an action taken by the agent $a \in \mathbb{A}$ has on the optimal choice of agent $0 \in \mathbb{A}$. In a similar manner, the random variable $L^{\varrho}$ measures the dependence of agents 0's best reply on his expectation about the population behavior.

The following assumption, MSI, restricts the functions describing the optimal actions of agents and is a natural generalization of the assumption made by Glaeser and Scheinkman in [18]. Later, in the context of particular examples, we will derive it from restrictions on the utility functions and interaction patterns. 
Definition 17. MSI holds for an ergodic system $\mathcal{E}$ satisfying Assumption 14, if the best reply function $h^{0}$ is Lipschitz continuous and if the constants $L^{a}$ can be chosen to satisfy

$$
\sum_{a \neq 0} L^{a}(\cdot) \leqslant \alpha<1 .
$$

MSI holds in strong form if we can choose constants $L^{a}$ and $L^{\varrho}$ such that

$$
\sup L^{\varrho}(\cdot)+\sup \sum_{a \neq 0} L^{a}(\cdot) \leqslant \alpha<1 .
$$

Inequalities (17) and (18) bound interactions across agents. MSI only involves the sensitivity of the optimal actions with respect to the individual actions, whereas MSI in strong form also bounds the sensitivity with respect to the anticipated distribution of actions. Our first result deals with existence and uniqueness of microscopic equilibria in homogeneous systems. Examples where Assumption 17 can be verified will be discussed in Section 4.2.

Proposition 18. If $\mathcal{E}$ is ergodic and MSI holds, then it has a unique and hence homogeneous microscopic equilibrium $g(\varrho, \cdot)$ with respect to every empirical distribution $\varrho$.

\subsubsection{Existence and uniqueness of equilibria in ergodic systems of social interactions}

Proposition 18 gives sufficient conditions for the existence of a homogeneous microscopic equilibrium for each $\varrho$. We now establish the existence of an equilibrium in an ergodic system that satisfy our MSI condition. Later we will use a weaker bound to prove existence results for a restricted class of systems of social interactions.

Theorem 19. If $\mathcal{E}$ is ergodic and has a homogeneous microscopic equilibrium $g(\varrho, \cdot)$ with respect to every $\varrho \in \mathcal{M}(X)$, then:

(i) The empirical distribution associated to the equilibrium action profile $g(\varrho, \cdot)$ exists, and is almost surely equal to $\mu[\varrho]$, the distribution of the random variable $g^{0}(\varrho, \cdot)$. That is,

$$
\lim _{n \rightarrow \infty} \frac{1}{\left|\mathbb{A}_{n}\right|} \sum_{a \in \mathbb{A}_{n}} \delta_{g^{a}(\varrho, J, \theta)}(\cdot)=\mu[\varrho] \quad \mathbb{P} \text {-a.s. }
$$

(ii) If $\mathcal{E}$ satisfies MSI, then it has a homogeneous equilibrium whose associated empirical distribution is almost surely independent of $(J, \theta)$.

(iii) If MSI holds in strong form, the equilibrium is unique.

A consequence of item (i) is that, if a homogeneous equilibrium exits for a purely local ergodic system then this equilibrium has necessarily an empirical distribution that is independent of the realization of the taste shocks or interaction patterns. The existence of a homogeneous equilibrium follows for instance from MSI. Hence in purely local ergodic system where MSI prevails, the empirical distribution of actions in equilibrium is (almost surely) independent of the particular realization of $(J, \theta)$. Although we defined equilibrium for such systems without requiring the existence of a well defined empirical distribution of actions, if MSI prevails and the system is ergodic we could now require, as part of the 
definition of equilibrium, that the empirical distribution of actions is well defined and independent of both the realized interaction pattern and the vector of taste shocks. If a system is homogeneous, but not necessarily ergodic, then the empirical distribution of actions would vary with $(J, \theta)$, but would be constant in each $\Omega_{v}$, the support of the distinct ergodic measures.

As far as we know there are no conditions on general systems of social interactions other than our MSI assumption which guarantee the existence of spatially homogeneous equilibria in random systems with locally interacting agents. The results in this section establish that in homogeneous ergodic systems the existence of equilibrium is guaranteed by MSI. Equilibrium is unique if MSI holds in strong form.

\subsubsection{Existence and uniqueness in ergodic systems with one-sided interactions}

We now consider a class of examples where existence and uniqueness of microscopic equilibria can be established under the assumption that the interaction between different agents is on average not too strong. To this end, we introduce the notion of one-sided interactions.

Definition 20. An ergodic system $\mathcal{E}=(\mathbb{A}, \mathbb{P}, X, U)$ has one sided interactions if:

(i) The random variables $\left(J^{a}, \theta^{a}\right)(a \in \mathbb{A})$ are distributed independently across agents.

(ii) The interaction pattern $J$ does not have cycles (almost surely.) That is, for almost all realizations, if there exists $a, b \in \mathbb{A}$ and sequence $a=a_{0}, a_{1}, a_{2}, \ldots, a_{n}, a_{n+1}=b \in$ A such that $J^{a_{i-1}, a_{i}} \neq 0$ for $i=1,2, \ldots, n$, then there is no sequence $\left(b_{i}\right)$ with $b_{0}=b$, and $b_{n+1}=a$ and $J^{b_{i-1}, b_{i}} \neq 0$ for all $i \in \mathbb{N}$.

Mean-field models with independently distributed $\theta^{a}$ 's constitute a particulary simple class of models with one-sided interactions. The following example illustrates the notion of one-sided interactions in a model with local interactions. More general systems with purely locally interacting agents where the interaction structure does not admit cycles are analyzed in, e.g., [10-12], and in a dynamic context with forward looking agents in [3].

Example 21. Let $\mathcal{E}=(\mathbb{Z}, \mathbb{P}, X, U)$ be a homogeneous system where the random variables $\left(J^{a}, \theta^{a}\right)(a \in \mathbb{A})$ are independent and where $\mathbb{P}\left[J^{a, b}=0\right.$ for all $\left.b \neq a+1\right]=1$. Then $\mathcal{E}$ is a system with one-sided interactions.

Existence and uniqueness results for ergodic systems with one-sided interactions can be established under a weaker bound on the strengths of social interactions. Specifically, we will use an AMSI condition. While MSI must hold for every realization of interaction patterns and taste shocks, AMSI must only hold in expected value. That is, AMSI holds if the interaction between different agents is on average not too strong.

Definition 22. Let $\mathcal{E}$ be an ergodic system satisfying Assumption 14.

(i) AMSI holds if the agents' best reply functions are Lipschitz continuous and if the constants $L^{a}$ can be chosen to satisfy

$$
\sum_{a \neq 0} \mathbb{E} L^{a}<1
$$


(ii) AMSI prevails in strong form, if the constants $L^{a}$ and $L^{\varrho}$ can be chosen to satisfy

$$
\mathbb{E} L^{\varrho}+\sum_{a \neq 0} \mathbb{E} L^{a}<1
$$

Again, AMSI only places bounds on the local interactions, whereas AMSI in strong form involves the global terms. For mean-field systems, since the utility an individual enjoys does not depend directly on the individual actions taken by his neighbors, we may choose $L^{a}=0$ and AMSI is trivially satisfied.

The next result states that in models with one-sided interactions AMSI guarantees the existence of homogeneous microscopic equilibria.

Proposition 23. Let $\mathcal{E}$ be an ergodic system with one-sided interactions. If AMSI holds, then $\mathcal{E}$ has a homogeneous microscopic equilibrium $g(\varrho, \cdot)$ with respect to every $\varrho \in \mathcal{M}(X)$.

We are now ready to state the main result of this section.

Theorem 24. Let $\mathcal{E}$ be an ergodic system with one-sided interactions.

(i) If AMSI holds, $\mathcal{E}$ has a homogeneous equilibrium $g(J, \theta)$ with associated empirical distribution almost surely independent of $(J, \theta)$.

(ii) If AMSI holds in strong form, then $\mathcal{E}$ has a unique equilibrium.

Remark 25. Let us consider again the mean-field model analyzed in [4] (Example 4). Since, in equilibrium, all agents share the same expectation about the behavior of their neighbors, $\mathbb{E}_{a} x^{b}=m$ for some $m \in[-1,1]$. Since the system is mean-field we may choose $L^{a}=0$, and AMSI is trivially satisfied. For mean-field systems with independently distributed $\theta^{a}$ 's our Theorem 24 may therefore by viewed as a version of Proposition 2 in [4]. If the dependence of an individual agent's optimal action on $\varrho$ is sufficiently strong, the system may have multiple equilibria. It admits a unique equilibrium whenever $\mathbb{E} L^{\varrho}<1$. Brock and Durlauf provide in [4] detailed intuition of how sufficient dependence generates multiplicity of equilibrium.

\subsubsection{Extension to the non-strictly concave case}

In the general case where utility functions are concave but not strictly concave with respect to own actions, our existence results apply to every homogeneous measurable selector $h=\left\{h^{a}\right\}_{a \in \mathbb{A}}$ of the agent's best reply correspondence defined by Eq. (15) above for some measurable selector $h^{0}$ of agent 0 's best reply correspondence. For instance, in analogy to Lemma 15, if there is a unique microscopic equilibrium once we fix a homogeneous measurable selector $h(\cdot, \varrho, J, \theta)$ of the conditional best reply correspondence, then this equilibrium is necessarily homogeneous. Also, the existence result stated in Proposition 18 applies to any homogeneous measurable selector $h$ of the best reply correspondence. If a selector $h(\cdot, \varrho, J, \theta)$ satisfies the MSI condition, it induces a homogeneous microscopic equilibrium with respect to any $\varrho$. An analogous modification of Theorem 19 also holds. In particular, the existence of a homogeneous measurable selector of the best reply correspondence satisfying MSI guarantees the existence of an equilibrium for an ergodic system. Similarly, 
for one-sided systems, existence holds if there is a measurable selector of the best reply correspondence satisfying AMSI. However, uniqueness is a more subtle problem, since our results would only imply uniqueness of equilibrium once we fix a measurable selection of the best reply correspondence and, in general, different equilibria may result from distinct selections.

\subsection{Examples of random systems with moderate social influence}

In this section we give some examples that illustrate our assumption of MSI. If the utility functions are sufficiently smooth, Assumptions 17 and 22 can be translated into conditions on marginal rates of substitution.

Example 26. Consider again the utility function specified in Example 5. It is easily seen that the (Average) MSI Assumption holds whenever $\beta_{2}$ is not too large. Thus, for a small enough $\beta_{2}$, the system has an equilibrium. It has a unique equilibrium if, in addition, $\beta_{1}$ is not too big.

Example 27. Consider the interaction pattern introduced in Example 1 and set $X=$ $[-1,1]$. Let $m(\varrho)=\int x \varrho(d x)$, and $m_{0}(J):=\frac{1}{M} \sum_{a \in N(0)} J^{a} x^{a}$. Suppose that there exists $u: X^{3} \times \Theta \rightarrow \mathbb{R}$ such that:

$$
U^{0}\left(x^{0},\left\{x^{a}\right\}_{a \neq 0}, \varrho, J^{0}, \theta^{0}\right)=u\left(x^{0}, m_{0}(J), m(\varrho), \theta^{0}\right) .
$$

We also assume that the map $\left(x^{0}, m_{0}, m\right) \mapsto u\left(x^{0}, m_{0}, m, \theta^{0}\right)$ is twice continuously differentiable with negative second derivative with respect to $x^{0}$. Further we assume that an agent's best reply is interior. The first order condition can be written as

$$
\frac{\partial u\left(x^{0}, m_{0}(J), m(\varrho), \theta^{0}\right)}{\partial x^{0}}=0 .
$$

Since $\frac{\partial^{2} u}{\partial\left(x^{0}\right)^{2}}<0$ it follows that the best reply of agent $0 \in \mathbb{A}$ is uniquely determined. Since $\mathbb{P}\left[J^{a}=0\right]=1$ for $a \notin N(0), L^{a}=0$ whenever $a \notin N(0)$. For $a \in N(0)$ it follows from $\mathbb{P}\left[\left|J^{a}\right| \leqslant 1\right]=1$ and from the implicit function theorem

$$
\left|\frac{\partial h^{0}\left(\left\{x^{a}\right\}_{a \neq a 0}, \varrho, J, \theta\right)}{\partial x^{a}}\right| \leqslant \frac{1}{M}\left|\frac{\frac{\partial^{2}}{\partial m_{0} \partial x^{0}} u\left(x^{0}, m_{0}(J), m(\varrho), \theta^{0}\right)}{\frac{\partial^{2}}{\partial\left(x^{0}\right)^{2}} u\left(x^{0}, m_{0}(J), m(\varrho), \theta^{0}\right)}\right| \quad \mathbb{P} \text {-a.s. }
$$

In particular, we can choose

$$
L^{a} \leqslant \sup _{x, \varrho} \frac{1}{M}\left|\frac{\frac{\partial^{2}}{\partial m_{0} \partial x^{0}} u\left(x^{0}, m_{0}(J), m(\varrho), \theta^{0}\right)}{\frac{\partial^{2}}{\partial\left(x^{0}\right)^{2}} u\left(x^{0}, m_{0}(J), m(\varrho), \theta^{0}\right)}\right| \quad \mathbb{P} \text {-a.s. }
$$


Thus MSI occurs if

$$
\sup _{x, \varrho}\left|\frac{\frac{\partial^{2}}{\partial m_{0} \partial x^{0}} u\left(x^{0}, m_{0}(J), m(\varrho), \theta^{0}\right)}{\frac{\partial^{2}}{\partial\left(x^{0}\right)^{2}} u\left(x^{0}, m_{0}(J), m(\varrho), \theta^{0}\right)}\right| \leqslant \alpha<1 \quad \text { P-a.s. }
$$

In this case, the system has an equilibrium. The equilibrium is unique if, for instance, the stronger condition

$$
\sup _{x, \varrho}\left|\frac{\frac{\partial^{2}}{\partial m_{0} \partial x^{0}} u\left(x^{0}, m_{0}(J), m(\varrho), \theta^{0}\right)}{\frac{\partial^{2}}{\partial\left(x^{0}\right)^{2}} u\left(x^{0}, m_{0}(J), m(\varrho), \theta^{0}\right)}\right|+\sup _{x, \varrho}\left|\frac{\partial^{2}}{\partial m \partial x^{0}} u\left(x^{0}, m_{a}(J), m(\varrho), \theta^{0}\right)\right| \leqslant \alpha
$$

holds almost surely for some $\alpha<1$. If the interaction is one-sided in the sense of Definition 20, we have an equilibrium whenever

$$
\mathbb{E}\left[\sup _{x, \varrho}\left|\frac{\frac{\partial^{2}}{\partial m_{0} \partial x^{0}} u\left(x^{0}, m_{0}(J), m(\varrho), \theta^{0}\right)}{\frac{\partial^{2}}{\partial\left(x^{0}\right)^{2}} u\left(x^{0}, m_{0}(J), m(\varrho), \theta^{0}\right)}\right|\right] \leqslant \alpha<1 .
$$

Let us now consider a generalization of the previous example.

Example 28. Consider again the interaction pattern described in Example 1. Let $X=$ $[-1,1]$ and assume that there exists a map $u: S \times X \times \Theta \rightarrow \mathbb{R}$ such that

$$
U^{0}\left(x, \varrho, J^{0}, \theta^{0}\right)=u\left(x^{0},\left\{J^{a} x^{a}\right\}_{a \neq 0}, m(\varrho), \theta^{0}\right) .
$$

Assume that the function $u$ is twice continuously differentiable with respect to both the actions $x^{b}$ taken by the agents and $m(\varrho)$ and that $\frac{\partial^{2} u}{\partial\left(x^{0}\right)^{2}}<0$. Using the same arguments as in Example 27 above, we see that moderate social interaction prevails if

$$
M \cdot \sup _{x, \varrho}\left|\frac{\frac{\partial}{\partial x^{a}} u\left(x^{0},\left\{J^{a} x^{a}\right\}_{a \neq 0}, m(\varrho), \theta^{0}\right)}{\frac{\partial^{2}}{\partial\left(x^{0}\right)^{2}} u\left(x^{0},\left\{J^{a} x^{a}\right\}_{a \neq 0}, m(\varrho), \theta^{0}\right)}\right| \leqslant \alpha<1 \quad \text { P-a.s. }
$$

\subsection{Weak interactions and social multipliers}

This section provides a rather informal discussion of social multipliers in random systems of social interactions. We extend the analysis in [18] to systems with a more general interaction structure and quantify the effect of a change in an exogenous parameter $p$ on the change in the average behavior throughout the entire population. To simplify our analysis we focus on systems with purely locally interacting agents.

Suppose utility functions $U^{a}(x, J, \theta, p)$ depend on some parameter $p \in \mathbb{R}, X \subset \mathbb{R}, U^{a}$ is sufficiently smooth and strongly concave with respect to $x^{a}$, and the agents' conditional optimal action is uniformly (on $a$ ) interior. In addition to our MSI condition we assume 
strategic complementarity that is, $\frac{\partial U^{a}\left(x, J^{a}, \theta^{a}, p\right)}{\partial x^{a} \partial x^{b}}>0$. Thus, an increase in his neighbor's action increases an agent's marginal utility of his own action.

As in [18], consider the mapping

$$
F(x, J, \theta, p)=x-g(x, J, \theta, p),
$$

where $g(x, \theta, J, p)$ denotes the configuration of conditional best replies, given the action profile $x$ and the triple $(\theta, J, p)$. The equilibrium action profile $g(J, \theta, p)$ solves

$$
F(g(J, \theta, p), J, \theta, p)=0 .
$$

Consider the linear operator $F_{x}(x, \theta, J, p)$ on $\mathbb{R}^{\mathbb{A}}$. MSI implies that this operator satisfies $\left\|I-F_{x}\right\|<1$. Thus, by Theorem II.1.2. in [33] the operator $F_{x}$ has a unique linear inverse $F_{x}^{-1}$ which is given by the Neumann series

$$
F_{x}^{-1}=I+\left(I-F_{x}\right)+\left(I-F_{x}\right)^{2}+\cdots=: I+H .
$$

Under these conditions the optimal action profile is a smooth function of $p$, and its derivative is given by

$$
\frac{\partial g(J, \theta, p)}{\partial p}=(I+H) \frac{\partial h(g, J, \theta, p)}{\partial p} .
$$

Strategic complementarity and MSI imply that $H \geqslant 0$. In this sense the effect on $g$ of changes in $p$ is an amplification of the effect on $g$ of the same change in $p$. The matrix with positive entries $H$ gives us a social multiplier.

\section{The structure of the equilibrium distribution}

We now present some properties of the structure of the distribution of equilibrium action profiles. To motivate our results, let us first examine the case where $\mathcal{E}$ is a mean-field system in the sense of our Definition 3, and the taste shocks are independent and identically distributed across agents with law $v$. Let $h^{a}\left(\theta^{a}, \varrho\right)=\arg \max _{x^{a}} U\left(x^{a}, \varrho, \theta^{a}\right)$ be the conditional optimal action of agent $a \in \mathbb{A}$, given his private taste shock $\theta^{a}$ and given his expectation $\varrho \in \mathcal{M}(X)$ about the distribution of behavior throughout the entire system. The law of the agent's optimal action is denoted by

$$
\gamma_{a}^{\varrho}(\cdot)=\int \delta_{h^{a}\left(\varrho, \theta^{a}\right)}(\cdot) v\left(d \theta^{a}\right) .
$$

The proof of the following proposition is trivial.

Proposition 29. Let $\mathcal{E}$ be a random mean field system and assume that the taste shocks are independent and identically distributed across agents. The law $\mu$ of an equilibrium action profile $g(\varrho, \theta)$ is of the form

$$
\mu(\cdot)=\prod_{a \in \mathbb{A}} \gamma_{a}^{\varrho}(\cdot) .
$$

In particular, $\mu$ is uniquely determined by the distribution of an agents' optimal actions. 
Let us now return to Föllmer's model of endogenous preference formation described in Section 3.2. In such a situation an agent's action reveals his taste shock since $h^{a}\left(\left\{x^{b}\right\}_{b \neq a}\right.$, $\left.\varrho, \theta^{a}\right)=\theta^{a}$. The conditional law $\gamma_{a}\left(\cdot ;\left\{x^{b}\right\}_{|b-a|=1}\right)$ of the equilibrium action of an agent $a \in \mathbb{A}$ given the actions of his neighbors does therefore take the form

$$
\gamma_{a}\left(\cdot ;\left\{x^{b}\right\}_{|b-a|=1}\right)=\pi_{a}\left(\cdot ;\left\{x^{b}\right\}_{|b-a|=1}\right) .
$$

Consider an outside observer who, in equilibrium, observes the actions of all the agents $b \neq a$. In Föllmer's model it is enough to observe the actions taken by the neighbors of $a \in \mathbb{A}$ in order to determine the distribution of this agent's equilibrium action. Our aim is to clarify to which extend this result carries over to more general models. The next example shows that when there are local interactions knowledge of a non-neighbor's action can help predict the action of an agent even after conditioning on the actions of all his neighbors. As a consequence, the conditional distribution of an agents' equilibrium action given the choices of all the other agents does not necessarily coincide with the law of his conditional best reply function.

Example 30. Agents are located on the one-dimensional integer lattice $\mathbb{Z}$ and the utility an agent $a \in \mathbb{A}$ enjoys depends on his own action, on the actions taken his neighbors, agent $a-1$ and agent $a+1$, and his private taste shock. The taste shocks $\theta^{a}(a \in \mathbb{A})$ are independent across agents and uniformly distributed on $[-2,0.5]$. Let $X=[0,1]$, and

$$
U\left(x^{a},\left\{x^{b}\right\}_{b \neq a}, \theta^{a}\right)=x^{a}\left(x^{a-1}+x^{a+1}-1+\theta^{a}\right) .
$$

The system is purely local, and hence by Proposition 13 it has an equilibrium. The conditional optimal action $h^{a}\left(\left\{x^{b}\right\}_{b \neq a}, J^{a}, \theta^{a}\right)$ of the agent $a \in \mathbb{A}$ satisfies

$$
h^{a}\left(\left\{x^{b}\right\}_{b \neq a}, J^{a}, \theta^{a}\right) \in\{0,1\} \quad \mathbb{P} \text {-a.s. }
$$

and the law $\gamma_{a}\left(\cdot ;\left\{x^{b}\right\}_{b \neq 0}\right)$ of the random variable $h^{a}\left(\left\{x^{b}\right\}_{b \neq a}, J^{a}, \theta^{a}\right)$ takes the from

$$
\gamma_{a}\left(1 ;\left\{x^{b}\right\}_{b \neq 0}\right)=\max \left\{\frac{2 x^{a+1}+2 x^{a-1}-1}{5}, 0\right\} .
$$

In particular, an agent $a \in \mathbb{A}$ never chooses action $x^{a}=1$ if both neighbors $b \in N(a)$ play $x^{b}=0$. Moreover, independently of his neighbors choices, the agent $a \in \mathbb{A}$ takes the action $x^{a}=0$ whenever $\theta^{a}<-1$. Consider now the event

$$
B:=\left\{\theta_{-2}<-1, \theta_{-1}>0, \theta_{0}>0, \theta_{1}>0, \theta_{2}<-1\right\} .
$$

The set $B$ has positive measure, and we may with no loss of generality assume that the equilibrium $g(J, \theta)=\left\{g^{a}(J, \theta)\right\}_{a \in \mathbb{A}}$ satisfies

$$
g^{ \pm 1}(J, \theta)=1 \quad \text { and } \quad g^{ \pm 2}(J, \theta)=0 \quad \text { on } B .
$$

In fact, such an equilibrium can be obtained from any other equilibrium via a suitable modification. Let us denote the law of the random variable $g(J, \theta)$ by $\mu$. Since $\mathbb{P}[B]>$ 0 we also have that $\mu\left(\left\{g^{ \pm 1}=1, g^{ \pm 2}=0\right\}\right)>0$ and so the conditional probability $\mu\left(g^{0}=1 \mid g^{ \pm 1}=1, g^{ \pm 2}=0\right)$ is well defined. Now recall that $\mu$ is concentrated on a set of 
equilibrium action profiles and that the agent $a=2$ is assumed to choose action $x^{2}=0$. Since, in equilibrium, agent $a=1$ chooses $x^{a}=1$ only if agent $a=0$ plays $x^{0}=1$,

$$
\mu\left(x^{0}=1 \mid g^{ \pm 1}=1, g^{ \pm 2}=0\right)=1 .
$$

For $x^{+1}=x^{-1}=1$, however, we have that

$$
\gamma_{0}\left(1 ;\left\{x^{b}\right\}_{b \neq 0}\right)=\frac{3}{5} \neq \mu\left(g^{0}=1 \mid g^{ \pm 1}=1, g^{ \pm 2}=0\right)=1 .
$$

Thus, the conditional marginal distribution of $\mu$ with respect to the actions $\left\{x^{b}\right\}_{b \neq a}$ is not described by the stochastic kernel $\gamma_{a}$. The fact that agent 2 chose action 0 and agent 1 chose action 1 means that agent 0 must have chosen action 1 . For otherwise agent 1 would have chosen action 0 . Hence knowledge of the action chosen by agent 2, who is not a neighbor of agent 0 tells an observer who knows the action of agent 1 something about the choice of agent 0 . This is because agents 0 and 2, though not neighbors of each other, have a common neighbor, agent 1 . On the other hand, no agent at distance bigger than 2 from agent 0 has a common neighbor with agent 0 . Hence knowledge of the actions taken by agents that are at distance bigger than 2 from agent 0 does not help predict the action of agent 0 , if the observer already knows the actions chosen of all agents that are located at distance 1 and 2. Theorem 32 below confirms that this intuition holds in general.

We have seen that in the special case of a pure mean field interaction, the law of the equilibrium action profile is uniquely determined by the stochastic kernels $\gamma^{a}(a \in \mathbb{A})$ on $X$ which describe the law of an agents' optimal action. Example 30 shows that in purely local systems, the law of the equilibrium action profile need not be determined by the laws of the agents conditional optimal actions, even if the taste shocks are independent and identically distributed across agents. In particular, given the equilibrium actions $x^{b}$ of all the agents $b \neq a$, the conditional distribution of agent $a$ 's action typically does not only depend on the actions taken by his neighbors.

The next theorem shows that the Markovian property that holds for Example 30 has a natural extension for purely local systems of social interactions with i.i.d. shocks and where no agents has neighbors at a distance more than a constant $M$. The intuition is again the same. Two agents at a distance more than $2 M$ are not neighbors and do not have a common neighbor. As a result, conditional on the actions taken by all the agents at distance at most $2 M$ from a given agent, knowledge of the actions of agents at further distance does not help predict that agent's action.

Definition 31. Let $\mathcal{E}$ be an ergodic systems of social interactions, and let $\mu$ be the distribution of a homogeneous equilibrium action profile $g$. $\mu$ has a Markov property of order $N \in \mathbb{N}$ if

$$
\mu\left(g^{0} \in \cdot \mid \mathcal{F}^{-0}\right)=\mu\left(g^{0} \in \cdot \mid \mathcal{F}_{N}\right) \quad \mu \text {-a.s. }
$$

where $\mathcal{F}^{-0}$ and $\mathcal{F}_{N}$ denote, respectively, the $\sigma$-fields generated by the random variables $\left\{g^{a}\right\}_{a \neq 0}$ and $\left\{g^{a}\right\}_{a \in B_{N}}$ and

$$
B_{N}:=\left\{b \in \mathbb{Z}^{d}: 1 \leqslant \max _{k=1, \ldots, d}\left|b^{k}\right| \leqslant N\right\} .
$$


The equilibrium distribution in the model specified in Section 3.2 has a Markov property of order 1. The following theorem states a general result about the structure of the distribution of equilibrium action profiles for purely local systems. The proof appears in Appendix B.

Theorem 32. If $\mathcal{E}$ is a purely local homogeneous system satisfying:

(i) There exists a constant $M<\infty$ such that $\mathbb{P}\left[J^{a, b}=0\right]=1$ whenever $\max _{k=1, \ldots, d} \mid a^{k}-$ $b^{k} \mid>M$. That is, the utility of an agent is not affected by actions of agents that are far enough.

(ii) The random variables $\left(J^{a}, \theta^{a}\right)$ are independent and identically distributed across agents.

Then the law of a homogeneous equilibrium action profile $g$ has a Markov property of order $2 M$.

For ergodic systems of social interactions where an agent's utility depends on both the actions taken by some neighbors and the average action throughout the whole population, we cannot expect a Markov property. Given a homogeneous equilibrium $g$, an outside observer knows the empirical distribution of all the agents' states once he observes all the actions $g^{a}$ with $a \neq 0$. This information is not available to him if he only observes the actions of a finite number of agents. However, if the observer knows the law $\mu$ of the equilibrium action profile, then he also knows the associated empirical distribution. In an ergodic system the latter is almost surely given by the one-dimensional marginal distributions of $\mu$. In this sense knowledge of $\mu$ implies knowledge of the empirical distribution of agents' actions in equilibrium. Thus, for models with local and global interactions, we have the following weaker Markov property.

Corollary 33. Let $\mathcal{E}$ be an ergodic system that satisfies assumption (i) and (ii) of Theorem 32 and let $\mu$ be the distribution of a homogeneous equilibrium g. Then $\mu$ has the following weak Markov property of order $2 M$ :

$$
\mu\left(g^{0} \in \cdot \mid \mathcal{F}^{-0}, \varrho\right)=\mu\left(g^{0} \in \cdot \mid \mathcal{F}_{B_{N}}, \varrho\right) \quad \mu \text { a.s. }
$$

where $\varrho$ denotes the empirical distribution associated to the equilibrium action profile $g$.

For non-purely local systems, the assumptions of the corollary allow dependence on actions of agents that are farther than $2 M$, but only through the empirical distribution of actions.

\section{Conclusion}

In this paper, we established existence and uniqueness results for systems of social interactions with an infinite number of agents in the presence of both local and global interactions. For purely local systems, continuity of the utility functions in the appropriate topology together with the usual convexity assumptions suffice. Note however that for systems with an 
infinite number of agents, continuity requires that the dependence of an agent's utility on other agents' actions decay sufficiently fast as the distance between agents increases.

In the presence of global interactions existence of an equilibrium seems to require further assumptions. We restricted our analysis to the case of spatially homogeneous systems. We first observed that we may view spatially homogeneous systems as mixtures of ergodic systems. We may thus think of a homogeneous system in two steps. Nature first picks an ergodic system using a distribution $\pi$, and then chooses an interaction pattern and a taste shock according to the distribution of the selected ergodic system.

If an ergodic system satisfies the assumption of moderate social interactions then existence of equilibrium obtains. In fact, we show the existence of an equilibrium where the empirical distribution of actions is always well defined and independent of the realized interaction pattern and taste shocks. For homogeneous systems this implies that the equilibrium distribution of actions only depends on the ergodic system chosen by nature. Furthermore, if the utility functions are strictly concave on own actions, a stronger form of MSI also delivers uniqueness of equilibrium for an ergodic system. For one-sided systems we obtain existence from the weaker assumption of average moderate social interactions. Uniqueness follows if we assume strict concavity and a stronger version of AMSI.

We also show that for purely local systems in which interactions have a maximum radius $M$, the law of the equilibrium profile is Markov of order $2 M$. An example shows that one cannot do better, and hence that we cannot describe equilibria via a family of stochastic kernels that specify the conditional distribution of actions by an agent, given the actions of his neighbors.

\section{Acknowledgments}

This research was supported by the Chaire Blaise Pascal de l'Etat et de la Région Ile de France, the Université Paris-Dauphine, the National Science Foundation through grant 0001647, the German Academic Exchange Service, and the DFG Research Center "Mathematics for Key Technologies" (MATHEON). We would like to thank two anonymous referees, an associate editor, Alberto Bisin, Hans Föllmer and seminar participants at several institutions for comments.

\section{Appendix A. Proof of the existence and uniqueness results}

In Appendix A we present the proofs of our existence and uniqueness results.

\section{A.1. Reduction to the ergodic case}

The analysis of homogeneous systems of social interactions can be reduced to the analysis of ergodic systems.

Proof of Proposition 11. Let $g$ be a homogeneous equilibrium for the homogeneous system $\mathcal{E}$. The ergodic theorem implies that the empirical distribution associated to the equi- 
librium action profile $g(J, \theta)$ exists almost surely and is, conditioned on $\Omega_{v}$ independent of $(J, \theta)$. This shows that the random variable $g_{v}$ on $\Omega_{v}$ given by

$$
g_{v}(J, \theta)=g(J, \theta) \quad \text { on } \quad \Omega_{v}
$$

defines a homogeneous equilibrium for $\mathcal{E}_{\eta}$. In order to show (ii) it is enough to show that $g$ is well defined, i.e., that

$$
\mathbb{P}\left[\bigcup_{v \neq \hat{v} \in \mathcal{M}_{0}} \Omega_{v} \cap \Omega_{\hat{v}}\right]=0 .
$$

This also follows from the ergodic theorem.

\section{A.2. Existence of microscopic equilibria}

Proof of Proposition 13. The utility functions are concave in the agents' own actions and continuous. Thus, the best reply correspondence $h$ is upper hemi-continuous. Since $X$ is compact and convex, the existence of a fixed point of the map $x=\left(x^{b}\right)_{b \in \mathbb{A}} \mapsto h(x, \varrho, J, \theta)$, i.e., a microscopic equilibrium associated to $\varrho$, follows from the Kakutani-Fan-Glicksberg fixed point theorem.

\section{A.3. Equilibria in ergodic systems}

This section proves existence and uniqueness results of equilibria in ergodic systems of random social interactions. In a first step we establish existence of homogeneous microscopic equilibria. We then use a fixed point argument to prove existence of an equilibrium.

\section{A.3.1. Existence and uniqueness of homogeneous microscopic equilibria}

We first prove our existence and uniqueness result for homogeneous microscopic equilibria for ergodic systems.

Proof of Lemma 15. Let $h$ be the best reply function, and $g(\varrho, \cdot)$ the unique microscopic equilibrium associated to $\varrho$. Then $g\left(\varrho, T^{b} J, T^{b} \theta\right)=\left\{g^{a}\left(\varrho, T^{b} J, T^{b} \theta\right)\right\}_{a \in \mathbb{A}}$ and $g(\varrho, J, \theta)=$ $\left\{g^{a}(\varrho, J, \theta)\right\}_{a \in \mathbb{A}}$ are the microscopic equilibrium configurations given $\left(T^{b} J\right.$, $\left.T^{b} \theta\right)$ and $(J, \theta)$, respectively. Homogeneity of the best reply function yields

$$
\begin{aligned}
g^{a-b}(\varrho, J, \theta) & \left.=h^{a-b}\left(\left\{g^{c}(\varrho, J, \theta)\right\}_{c \neq a-b}, \varrho, J, \theta\right\}\right) \\
& \left.=h^{a}\left(\left\{g^{c-b}(\varrho, J, \theta)\right\}_{c \neq a}, \varrho, T^{b} J, T^{b} \theta\right\}\right)
\end{aligned}
$$

almost surely. Thus, given $\left(T^{b} J, T^{b} \theta\right)$, if player $a$ chooses the action $g^{a-b}(\varrho, J, \theta)$ then no agent has an incentive to deviate. This shows that $\left\{g^{a-b}(\varrho, J, \theta)\right\}_{a \in \mathbb{A}}$ is an equilibrium action profile for $\left(T^{b} J, T^{b} \theta\right)$, and so uniqueness of equilibria implies homogeneity:

$$
g^{0}\left(\varrho, T^{b} J, T^{b} \theta\right)=g^{-b}(\varrho, J, \theta) \quad \mathbb{P} \text {-a.s. }
$$


Proof of Proposition 18. Let us fix $\varrho \in \mathcal{M}(X)$ as well as an interaction pattern $J$ and a configuration of taste shocks $\theta$. For a given action profile $x \in S$ we put

$$
h(x, \varrho, J, \theta)=\left\{h^{a}\left(\left\{x^{b}\right\}_{b \neq a}, \varrho, J^{a}, \theta^{a}\right)\right\}_{a \in \mathbb{A}} .
$$

The existence of microscopic equilibria with respect to $\varrho$ has already been established. In order to show uniqueness of equilibria in homogeneous systems in which MSI prevails, we fix configurations $x, y \in S$. Under MSI we have $\mathbb{P}$-a.s. that

$$
\begin{aligned}
& \left|h^{0}\left(\left\{x^{a}\right\}_{a \neq 0}, \varrho, J, \theta\right)-h^{0}\left(\left\{y^{a}\right\}_{a \neq 0}, \varrho, J, \theta\right)\right| \\
& \quad \leqslant \sum_{a \neq 0} L^{a}\left(J^{0}, \theta^{0}\right)\left|x^{a}-y^{a}\right| \leqslant \alpha \max _{a \in \mathbb{A}}\left|x^{a}-y^{a}\right| .
\end{aligned}
$$

Thus, by homogeneity of the best reply function, for $x \neq y$ we have for almost all interaction patterns and taste shocks that

$$
\max _{a \in \mathbb{A}}\left|h^{a}\left(\left\{x^{b}\right\}_{b \neq a}, \varrho, J, \theta\right)-h^{a}\left(\left\{y^{b}\right\}_{b \neq a}, \varrho, J, \theta\right)\right|<\max _{a \in \mathbb{A}}\left|x^{a}-y^{a}\right| .
$$

This shows that the map $x \mapsto h(x, \varrho, J, \theta)$ has almost surely at most one fixed point. Spatial homogeneity of the equilibrium follows from Lemma 15.

\section{A.3.2. Existence and uniqueness of equilibria}

This section contains the proofs of existence and uniqueness results for equilibria in ergodic systems of social interactions. In a first step we prove that distribution $\mu[\varrho]$ of an agent's actions $g^{a}(\varrho, \cdot)$ depends in a Lipschitz continuous manner on the perceived empirical distribution $\varrho$ if the interaction between different agents is not too strong. We then show that the map $\varrho \mapsto \mu[\varrho]$ has a fixed point.

Lemma A.1. Let $\mathcal{E}$ be an ergodic random system and let $g(\varrho, \cdot)$ be a homogeneous microscopic equilibrium with respect to $\varrho$. If MSI occurs, then the map $\varrho \mapsto g^{0}(\varrho, \cdot)$ is almost surely Lipschitz continuous. That is, there exists a constant $L<\infty$ such that

$$
\left|g^{0}(\hat{\varrho}, \cdot)-g^{0}(\tilde{\varrho}, \cdot)\right| \leqslant L d_{V}(\underline{\varrho}, \tilde{\varrho}) \quad \mathbb{P}-a . s .
$$

If MSI holds in strong form, the constant L in (A.1) is strictly less than 1.

Proof. Since $\left\{g^{a}(\varrho, J, \theta)\right\}_{a \in \mathbb{A}}$ is an equilibrium with respect to $\varrho$, we have

$$
g^{a}(\varrho, J, \theta)=h^{a}\left(\left\{g^{b}(\varrho, J, \theta)\right\}_{a \neq b}, \varrho, J, \theta\right) .
$$

Thus, for $\hat{\varrho}, \underline{\varrho} \in \mathcal{M}(X)$ and $a \in \mathbb{A}$,] on a set of full measure

$$
\begin{aligned}
& \left|g^{0}(\hat{\varrho}, J, \theta)-g^{0}(\tilde{\varrho}, J, \theta)\right| \\
& \leqslant\left|h^{0}\left(\left\{g^{a}(\underline{\varrho}, J, \theta)\right\}_{a \neq 0}, \hat{\varrho}, J, \theta\right)-h^{0}\left(\left\{g^{a}(\varrho \hat{\varrho}, J, \theta)\right\}_{a \neq 0}, \tilde{\varrho}, J, \theta\right)\right| \\
& +\left|h^{0}\left(\left\{g^{a}(\hat{\varrho}, J, \theta)\right\}_{a \neq 0}, \tilde{\varrho}, J, \theta\right)-h^{0}\left(\left\{g^{0}(\tilde{\varrho}, J, \theta)\right\}_{a \neq 0}, \tilde{\varrho}, J, \theta\right)\right| \\
& \leqslant L^{\varrho}\left(\theta^{0}\right) d_{V}(\hat{\varrho}, \tilde{\varrho})+\sum_{a \neq 0} L^{a}\left(J^{0}, \theta^{0}\right)\left|g^{a}(\varrho, J, \theta)-g^{a}(\varrho, J, \theta)\right|
\end{aligned}
$$


because an agent's best reply function is Lipschitz continuous. Homogeneity of the microscopic equilibrium yields

$$
\begin{aligned}
& \left|g^{0}(\underline{\varrho}, J, \theta)-g^{0}(\tilde{\varrho}, J, \theta)\right| \\
& \quad \leqslant L^{\varrho}\left(\theta^{0}\right) d_{V}(\hat{\varrho}, \tilde{\varrho})+\sum_{a \neq 0} L^{a}\left(J^{0}, \theta^{0}\right)\left|g^{0}\left(\hat{\varrho}, T^{a} J, T^{a} \theta\right)-g^{0}\left(\tilde{\varrho}, T^{a} J, T^{a} \theta\right)\right|,
\end{aligned}
$$

and so

$$
\begin{aligned}
& \sup \left|g^{0}(\hat{\varrho}, \cdot)-g^{0}(\tilde{\varrho}, \cdot)\right| \\
& \quad \leqslant d_{V}(\hat{\varrho}, \tilde{\varrho}) \sup L^{\varrho}(\cdot)+\sup \left|g^{0}(\hat{\varrho}, \cdot)-g^{0}(\varrho \underline{\varrho}, \cdot)\right| \sup \sum_{a \neq 0} L^{a}(\cdot) .
\end{aligned}
$$

Thus, the MSI condition yields

$$
\left|g^{0}(\hat{\varrho}, \cdot)-g^{0}(\tilde{\varrho}, \cdot)\right| \leqslant \frac{\sup L^{\varrho}(\cdot)}{1-\sup \sum_{a \neq 0} L^{a}(\cdot)} d_{V}(\hat{\varrho}, \tilde{\varrho}) \quad \mathbb{P} \text {-a.s. }
$$

We now show that the empirical distribution that obtains in a homogeneous microscopic equilibrium associated with a distribution $\varrho$ is almost surely independent of both the realized interaction pattern and the actual configuration of taste shocks, and that it varies continuously with $\varrho$ if MSI prevails.

Lemma A.2. Let $\mathcal{E}$ be an ergodic system. Denote by $g(\varrho, \cdot)$ a homogeneous microscopic equilibrium associated to $\varrho \in \mathcal{M}(X)$ and by $\mu[\varrho]$ the law of the random variable $g^{0}(\varrho, \cdot)$.

(i) The empirical distribution associated to the equilibrium action profile $g(\varrho, J, \theta)$ exists and is almost surely independent of the realized interaction pattern and the configuration of taste shocks. That is,

$$
\lim _{n \rightarrow \infty} \frac{1}{\left|\mathbb{A}_{n}\right|} \sum_{a \in \mathbb{A}_{n}} \delta_{g^{a}(\varrho, J, \theta)}(\cdot)=\mu[\varrho] \quad \mathbb{P}-\text { a.s. }
$$

(ii) If MSI holds, then the mapping $\varrho \mapsto \mu[\varrho]$ is continuous and has a fixed-point. The fixed point is unique if MSI holds in strong form.

Proof. Since the map $g^{0}(\varrho, \cdot)$ is bounded and measurable and because the sequence of random variables $\left\{\left(J^{a}, \theta^{a}\right)\right\}_{a \in \mathbb{A}}$ is stationary and ergodic, our first assertion follows from Birkhoff's ergodic theorem:

$$
\begin{aligned}
& \lim _{n \rightarrow \infty} \frac{1}{\left|\mathbb{A}_{n}\right|} \sum_{a \in \mathbb{A}_{n}} \delta_{g^{a}(\varrho, J, \theta)}(\cdot) \\
& =\lim _{n \rightarrow \infty} \frac{1}{\left|\mathbb{A}_{n}\right|} \sum_{a \in \mathbb{A}_{n}} \delta_{g^{0}\left(\varrho, T^{a} J, T^{a} \theta\right)}(\cdot) \\
& =\mu[\varrho] \quad \mathbb{P} \text {-a.s. }
\end{aligned}
$$


In order to establish (ii), we fix a bounded Lipschitz continuous function $f: X \rightarrow \mathbb{R}$. The ergodic theorem yields

$$
\int f d \mu[\varrho]=\int f \circ g^{0}(\varrho, J, \theta) d \mathbb{P} .
$$

Thus, in view of Lemma A.1 we have

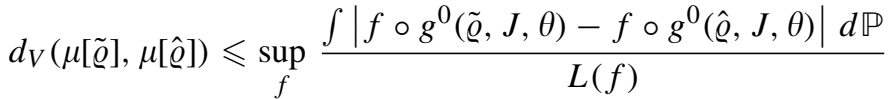

$$
\begin{aligned}
& \leqslant \int^{f}\left|g^{0}(\tilde{\varrho}, J, \theta)-g^{0}(\underline{\varrho}, J, \theta)\right| d \mathbb{P} \leqslant L d_{V}(\underline{\varrho}, \hat{\varrho})
\end{aligned}
$$

whenever MSI prevails. Thus, the map $\varrho \mapsto \mu[\varrho]$ is continuous. Since $\left(\mathcal{M}(X), d_{V}\right)$ is a compact metric space, our assertion follows from Brower's fixed point theorem. If MSI holds in strong form, the map $\varrho \mapsto \mu[\varrho]$ is a contraction. In this case the fixed point is unique.

We are now ready to prove the main results of this paper.

Proof of Theorem 19. Let $g(\varrho, \cdot)$ be a homogeneous equilibrium with respect to $\varrho$. By Lemma A. 2 that there exists $\varrho^{*} \in \mathcal{M}(X)$ such that

$$
\varrho^{*}=\lim _{n \rightarrow \infty} \frac{1}{\left|\mathbb{A}_{n}\right|} \sum_{a \in \mathbb{A}_{n}} \delta_{g^{a}\left(\varrho^{*}, J, \theta\right)}(\cdot) .
$$

Thus the $\varrho^{*}$-equilibrium action profile $\left\{g^{a}\left(\varrho^{*}, J, \theta\right)\right\}_{a \in \mathbb{A}}$ is almost surely an equilibrium in the sense of our Definition 7. The uniqueness claim also follows from Lemma A.2.

\section{A.4. Equilibria in ergodic systems with one-sided interactions}

This section contains the proofs of existence and uniqueness results for equilibria in ergodic systems with one-sided interactions. We modify arguments that have previously been applied in [3] to prove existence of equilibria in dynamic models of social interactions. Define an operator $V$ on the class of all bounded measurable functions from $\Omega$ to $X$ by

$$
\begin{aligned}
& V f(J, \theta)=\{(V f)(J, \theta)\}_{a \in \mathbb{A}} \text { with } \\
& \quad(V f)(J, \theta):=h^{0}\left(\left\{f\left(T^{a} J, T^{a} \theta\right)\right\}_{a \neq 0}, \varrho, J^{0}, \theta^{0}\right) .
\end{aligned}
$$

Every fixed point $f(\varrho, \cdot)$ of $V$ gives rise to a homogeneous equilibrium with respect to $\varrho$. Indeed, the best reply functions satisfy the homogeneity conditions

$$
h^{a}\left(\left\{x^{c}\right\}_{c \neq a}, \varrho, J, \theta\right)=h^{0}\left(\left\{x^{c-a}\right\}_{c \neq a}, \varrho, T^{a} J, T^{a} \theta\right) .
$$

Thus, with $y^{a}(\varrho, J, \theta):=f\left(\varrho, T^{a} J, T^{a} \theta\right)$ we obtain

$$
\begin{aligned}
y^{a}(\varrho, J, \theta) & =V f\left(\varrho, T^{a} J, T^{a} \theta\right) \\
& =h^{0}\left(\left\{f\left(\varrho, T^{a+b} J, T^{a+b} \theta\right)\right\}_{b \neq 0}, \varrho, T^{a} J, T^{a} \theta\right) \\
& =h^{0}\left(\left\{y^{b-a}(\varrho, J, \theta)\right\}_{b \neq 0}, \varrho, T^{a} J, T^{a} \theta\right) \\
& =h^{a}\left(\left\{y^{b}(\varrho, J, \theta)\right\}_{b \neq a}, \varrho, J, \theta\right) .
\end{aligned}
$$


In particular, a homogeneous configuration $g(\varrho, \cdot)$ is a microscopic equilibrium with respect to $\varrho$ if and only if $g(\varrho, \cdot)$ is a fixed point of the operator $V$. In order to prove Proposition 23 it is thus enough to show that $V$ has a unique fixed point. For this, we introduce the interaction matrix $\hat{L}=\left(L^{a, b}\right)_{a, b \in \mathbb{A}}$ with entries $L^{a, b}:=L^{b-a} \circ T^{a}$. The quantity $L^{a, b}$ measures the dependence of the best reply of the agent $a$ on the action taken by agent $b$. Observe that $L^{0, a}=L^{a}$ so that homogeneity along with AMSI implies $\sum_{b \in \mathbb{A}} \mathbb{E} L^{a, b}=\sum_{b \in \mathbb{A}} \mathbb{E} L^{b}<1$ for all $a \in \mathbb{A}$.

The following lemma turns out to the key to the proof of our existence and uniqueness result for one-sided interaction.

Lemma A.3. Let $L_{n}=\left(L_{n}^{a, b}\right)$ be the nth power of the matrix $L=\left(L^{a, b}\right)$. Then

$$
\lim _{n \rightarrow \infty} \sum_{a \in \mathbb{A}} \mathbb{E} L_{n}^{0, a}=0
$$

whenever the assumption of Theorem 24 are satisfied.

Proof. Let $\mathcal{E}$ be a homogeneous system with one-sided interactions. The random variables $L^{a, b}$ depend on the entire interaction pattern $J$ and on the vector of taste shocks $\theta$ only through $J^{a}$ and $\theta^{a}$. In particular, the random variables $L^{a, b}$ and $L^{c, d}$ are independent for $a \neq c$ because the interaction patterns have no circles. Thus

$$
\mathbb{E}\left[L^{a_{1}, a_{2}} L^{a_{2}, a_{3}} \cdots L^{a_{n-1}, a_{n}}\right]=\prod_{i=1}^{n-1} \mathbb{E} L^{a_{i}, a_{i+1}}
$$

for any sequence $a_{1}, a_{2}, \ldots, a_{n}$ and all $n \in \mathbb{N}$. By homogeneity of the best reply function, $\mathbb{E} L^{0, a}=\mathbb{E} L^{b, a+b}$, and so an induction argument shows

$$
\begin{aligned}
\sum_{a \in \mathbb{A}} \mathbb{E} L_{n+1}^{0, a} & =\sum_{a, b \in \mathbb{A}} \mathbb{E}\left[L^{0, a} L_{n}^{a, b}\right]=\sum_{a \in \mathbb{A}} \mathbb{E} L^{0, a} \sum_{b \in \mathbb{A}} \mathbb{E} L_{n}^{a, b} \\
& \leqslant\left(\sum_{a \in \mathbb{A}} \mathbb{E} L^{0, a}\right)\left(\sum_{a \in \mathbb{A}} \mathbb{E} L^{0, a}\right)^{n} \leqslant\left(\sum_{a \in \mathbb{A}} \mathbb{E} L^{0, a}\right)^{n+1} .
\end{aligned}
$$

Thus, the assertion follows from the AMSI assumption.

We now prove existence and uniqueness of homogeneous microscopic equilibria.

Proof of Proposition 23. For any bounded measurable functions $f, \hat{f}$ our AMSI assumption yields

$$
|V f-V \hat{f}| \leqslant \sum_{a \in \mathbb{A}} L^{0, a}\left|f \circ T^{a}-\hat{f} \circ T^{a}\right| \quad \mathbb{P} \text {-a.s. }
$$

By iteration we obtain

$$
\left|V^{2} f-V^{2} \hat{f}\right| \leqslant \sum_{a \in \mathbb{A}} \sum_{b \in \mathbb{A}} L^{0, a} L^{a, b}\left|f \circ T^{a+b}-\hat{f} \circ T^{a+b}\right| \quad \mathbb{P} \text {-a.s. }
$$


and for any $n \in \mathbb{N}$

$$
\left|V^{n} f-V^{n} \hat{f}\right| \leqslant \sum_{b \in \mathbb{A}} L_{n}^{a, b}\left(\|f\|_{\infty}+\|\hat{f}\|_{\infty}\right) \quad \mathbb{P} \text {-a.s. }
$$

Thus, Lemma A.3 yields

$$
\lim _{n \rightarrow \infty} \mathbb{E}\left|V^{n} f-V^{n} \hat{f}\right| \leqslant \lim _{n \rightarrow \infty}\left(\sum_{a \in \mathbb{A}} \mathbb{E} L^{0, a}\right)^{n}\left(\|f\|_{\infty}+\|\hat{f}\|_{\infty}\right)=0 .
$$

This shows that the sequence $\left\{V^{n} f\right\}_{n \in \mathbb{N}}$ is a Cauchy sequence in $L^{1}(\Omega, \mathbb{P})$. Since the space $L^{1}(\Omega, \mathbb{P})$ is complete and because of (A.6), the sequence $\left\{V^{n} f\right\}_{n \in \mathbb{N}}$ converges in $L^{1}(\Omega, \mathbb{P})$ to a random variable $f(\varrho, \cdot)$ that does not depend on the starting point $f$. Since $L^{1}$-convergence implies convergence in probability, for every $\varepsilon>0$ there exists $N_{0} \in \mathbb{N}$ such that

$$
\mathbb{P}\left[\left|V^{n} f(\varrho, \cdot)-f(\varrho, \cdot)\right| \leqslant \frac{\varepsilon}{2}\right] \geqslant 1-\frac{\varepsilon}{2} \quad \text { for all } n \geqslant N_{0} .
$$

Lipschitz continuity of the best reply function $h^{0}$ guarantees that $N_{0}$ can be chosen to satisfy

$$
\mathbb{P}\left[\left|V V^{n-1} f(\varrho, \cdot)-V f(\varrho, \cdot)\right| \leqslant \frac{\varepsilon}{2}\right] \geqslant 1-\frac{\varepsilon}{2} \text { for all } n \geqslant N_{0} .
$$

Combining (A.7) and (A.8) we obtain

$$
\mathbb{P}\left[\left\{\left|V^{n} f(\varrho, \cdot)-f(\varrho, \cdot)\right| \leqslant \frac{\varepsilon}{2}\right\} \cap\left\{\left|V V^{n-1} f(\varrho, \cdot)-V f(\varrho, \cdot)\right| \leqslant \frac{\varepsilon}{2}\right\}\right] \geqslant 1-\varepsilon .
$$

Since

$$
\begin{aligned}
& \left\{\left|V^{n} f(\varrho, \cdot)-f(\varrho, \cdot)\right| \leqslant \frac{\varepsilon}{2}\right\} \cap\left\{\left|V V^{n-1} f(\varrho, \cdot)-V f(\varrho, \cdot)\right| \leqslant \frac{\varepsilon}{2}\right\} \\
& \quad \subset\{|V f(\varrho, \cdot)-f(\varrho, \cdot)| \leqslant \varepsilon\}
\end{aligned}
$$

this shows that

$$
\mathbb{P}[|V f(\varrho, \cdot)-f(\varrho, \cdot)| \leqslant \varepsilon] \geqslant 1-\varepsilon \text { for any } \varepsilon>0 .
$$

Thus,

$$
\mathbb{P}[V f(\varrho, \cdot)=f(\varrho, \cdot)]=1,
$$

and so $f(\varrho, \cdot)$ is a microscopic equilibrium. In order to establish uniqueness recall that convergence in probability implies almost sure convergence along a suitable subsequence. For any fixed point $f^{*}(\varrho, \cdot)$ of the operator $V$ we can thus find a subsequence $\left\{n_{k}\right\}_{k \in \mathbb{N}}$ such that

$$
\lim _{k \rightarrow \infty} V^{n_{k}} f^{*}(\varrho, \cdot)=f(\varrho, \cdot) \quad \mathbb{P} \text {-a.s. }
$$

Since $f^{*}(\varrho, \cdot)$ is a fixed point, $f^{*}(\varrho, \cdot)=V^{n_{k}} f^{*}(\varrho, \cdot)$ almost surely, and so $\mathbb{P}[f(\varrho, \cdot)=$ $\left.f^{*}(\varrho, \cdot)\right]=1$. 
The next result shows that, in a microscopic equilibrium, an agent's actions depends, on average, in a Lipschitz continuous manner on the perceived empirical distribution $\varrho$.

Lemma A.4. Let $\mathcal{E}$ be an ergodic system with one-sided interactions and let $g(\varrho, \cdot)$ be a homogeneous microscopic equilibrium with respect to $\varrho$. If AMSI holds, the maps $\varrho \mapsto$ $g^{a}(\varrho, \cdot)$ are Lipschitz continuous on average:

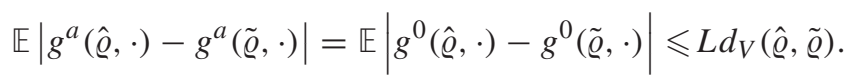

If AMSI prevails in strong form, the constant L in (A.9) is strictly less than 1.

Proof. Homogeneity of the system $\mathcal{E}$ and the equilibrium $g$ yields

$$
\mathbb{E}\left|g^{0}(\hat{\varrho}, \cdot)-g^{0}(\tilde{\varrho}, \cdot)\right|=\mathbb{E}\left|g^{a}(\hat{\varrho}, \cdot)-g^{a}(\tilde{\varrho}, \cdot)\right| \quad \text { for all } a \in \mathbb{A} \text {. }
$$

In view of (A.3) we have

$$
\mathbb{E}\left|g^{0}(\hat{\varrho}, \cdot)-g^{0}(\tilde{\varrho}, \cdot)\right| \leqslant \mathbb{E} L^{\varrho} d_{V}(\hat{\varrho}, \tilde{\varrho})+\mathbb{E}\left[\sum_{a \neq 0} L^{a}(\cdot)\left|g^{a}(\hat{\varrho}, \cdot)-g^{a}(\tilde{\varrho}, \cdot)\right|\right] \text {. }
$$

Note that the random variable $L^{a}$ depends on $\left(J^{0}, \theta^{0}\right)$ whereas the random variable $g^{a}(\varrho, \cdot)$ depends on the realizations of the interaction patterns $J^{b}$ and taste shocks $\theta^{b}$ of the agents $b \in \mathbb{A}$ with whom the agent $a$ is directly or indirectly linked. For a system $\mathcal{E}$ with one-sided interactions this means that the random variables $L^{a}$ and $g^{a}(\varrho, \cdot)$ are independent. Thus

$$
\mathbb{E}\left|g^{0}(\underline{\varrho}, \cdot)-g^{0}(\tilde{\varrho}, \cdot)\right| \leqslant \mathbb{E} L^{\varrho} d_{V}(\varrho \underline{\varrho}, \tilde{\varrho})+\sum_{a \neq 0} \mathbb{E} L^{a} \mathbb{E}\left|g^{a}(\hat{\varrho}, \cdot)-g^{a}(\tilde{\varrho}, \cdot)\right| .
$$

Thus, (A.10) along with our AMSI condition shows that

$$
\mathbb{E}\left|g^{0}(\varrho, \cdot)-g^{0}(\varrho, \cdot)\right| \leqslant \frac{\mathbb{E} L^{\varrho}}{1-\sum_{a \neq 0} \mathbb{E} L^{a}} .
$$

This proves our assertion.

We are now ready to prove the main result of Section 4.1.6.

Proof of Theorem 24. Let $g(\varrho, \cdot)$ be a homogeneous microscopic equilibrium. Proceeding as in the proof of Lemma A.2 we can show that the empirical distribution $\mu[\varrho]$ associated to the actions profile $g(\varrho, \cdot)$ exists almost surely, that the map $\varrho \mapsto \mu[\varrho]$ is Lipschitz continuous (a contraction) if AMSI holds (in strong form). Existence and uniqueness of equilibria follow as in the proof of Theorem 19.

\section{Appendix B. Proof of the Markov property}

In Appendix B we prove the Markov property of the distribution $\mu$ of an equilibrium action profile $g$. For notational convenience we restrict ourselves to the case where the 
agents are located on the one-dimensional integer lattice, i.e., to $\mathbb{A}=\mathbb{Z}$, and assume a nearest neighbor interaction, i.e., $M=1$ and $J^{a, a \pm 1}=1, J^{a, b}=0$ otherwise. The general case follows from similar considerations.

Proof of Theorem 32. For a given action profile $x^{-0}=\left\{x^{a}\right\}_{a \neq 0}$ let

$$
\hat{x}^{-0}=\left\{x^{-2}, x^{-1}, x^{+1}, x^{+2}\right\}
$$

be its projection on the actions taken by the agents $a \in\{-2,-1,1,2\}$, and for $B=$ $\left\{\left\{x^{a}\right\}_{a \neq 0}: x^{a} \in X\right\}$ we put $\hat{B}:=\left\{\hat{x}^{-0}: x^{-0} \in B\right\}$. We need to prove that $\mu$ satisfies

$$
\mu(A \mid B)=\mu(A \mid \hat{B}) \quad \text { for any measurable } A \subset X \text { and all } B \text { with } \quad \mu(B)>0 .
$$

To this end, we proceed in three steps.

(i) Given a set $B$ of possible equilibrium actions $g^{-0}$ of all the other agents $a \neq 0$, we have additional information about the equilibrium action $x^{0}$ of the agent 0 . Since $\mu$ is concentrated on the set of all equilibrium action profiles, we know that $x^{0}$ must stabilize the best replies of agent 0 's neighbors for at least some action profile $g^{-0} \in B$. More precisely, the agent 0 necessarily takes an action from the set

$$
\begin{aligned}
G(B):= & \left\{x^{0} \in X: \text { there exists } \theta=\left\{\theta^{a}\right\}_{a \in \mathbb{A}}\right. \text { such that the equilibrium } \\
& \text { actions } \left.g^{-0}(\theta) \text { satisfy } g^{-0} \in B \text { and } g^{ \pm 1}=h^{0}\left(\theta^{ \pm 1}, x^{0}, g^{ \pm 2}\right)\right\} .
\end{aligned}
$$

Thus, $\mu(A \mid B)=\mathbb{P}\left[g^{0} \in A \mid g^{0} \in G(B)\right]$. Since $\mu(B)>0$ implies $\mathbb{P}\left[g^{0} \in G(B)\right]>$ 0 , Baey's formula yields

$$
\mu(A \mid B)=\frac{\mathbb{P}\left[g^{0} \in A \cap G(B)\right]}{\mathbb{P}\left[g^{0} \in G(B)\right]} .
$$

If we only observe the actions of the agents $b \in\{-2,-1,1,2\}$, we have, in principle, less information. In principle we only know that player 0's choice must stabilize his neighbors' best replies given some equilibrium actions $y^{-0}$ such that $\hat{y}^{-0} \in \hat{B}$. Thus, we only know that the agent chose an action some the set

$$
\begin{aligned}
\hat{G}(B):= & \left\{x^{0} \in X: \text { there exists } \theta=\left\{\theta^{a}\right\}_{a \in \mathbb{A}}\right. \text { such that the equilibrium } \\
& \text { actions } \left.g^{-0}(\theta) \text { satisfy } \hat{g}^{-0}(\theta) \in \hat{B} \text { and } g^{ \pm 1}=h^{0}\left(\theta^{ \pm 1}, x^{0}, g^{ \pm 2}\right)\right\} .
\end{aligned}
$$

By analogy to (B.1) this yields

$$
\mu(A \mid \hat{B})=\frac{\mathbb{P}\left[g^{0} \in A \cap \hat{G}(B)\right]}{\mathbb{P}\left[g^{0} \in \hat{G}(B)\right]} .
$$

(ii) Let us denote by $v$ the law of the of the random variables $\theta^{a}$ and introduce the set

$$
H(B):=\left\{\theta=\left\{\theta^{a}\right\}_{a \in \mathbb{A}}: g^{-0}(\theta) \in B\right\}
$$


of all configurations of taste shocks which are compatible with the fact that the equilibrium actions $g^{-0}$ belong to the set $B$. Independence of the random variables $\theta^{a}$ $(a \in \mathbb{A})$ yields

$$
\begin{aligned}
\mathbb{P}\left[g^{0} \in G(B)\right] & =\int \mathbf{1}_{H(B)}\left(\left\{\theta^{a}\right\}_{a \in \mathbb{A}}\right) \prod_{a \in \mathbb{A}} v\left(d \theta^{a}\right) \\
& =\iint \mathbf{1}_{H(B)}\left(\theta^{ \pm 1},\left\{\theta^{b}\right\}_{b \neq \pm 1}\right) \prod_{b \neq \pm 1} v\left(d \theta^{b}\right) \prod_{a= \pm 1} v\left(d \theta^{a}\right) \\
& =\int \mathbb{P}\left[g^{0} \in G(B) \mid \theta^{ \pm 1}\right] \prod_{a= \pm 1} v\left(d \theta^{a}\right)
\end{aligned}
$$

and the following representation of the conditional probability of $\left\{g^{0} \in G(B)\right\}$, given $\left(\theta^{-1}, \theta^{-1}\right)$ :

$$
\begin{aligned}
& \mathbb{P}\left[g^{0} \in G(B) \mid \theta^{ \pm 1}\right] \\
& =v\left[\theta^{0}: \text { there exists } y^{-0} \in B \text { such that with } g^{0}=h^{0}\left(\theta^{0}, y^{ \pm 1}\right)\right. \text { we have } \\
& \left.\quad y^{ \pm 1}=h^{ \pm 1}\left(\theta^{ \pm 1}, g^{0}, y^{ \pm 2}\right) .\right]
\end{aligned}
$$

We now consider the quantity $\mathbb{P}\left[g^{0} \in \hat{G}(B)\right]$. By analogy to (B.3) we introduce the set

$$
\hat{H}(B):=\left\{\theta=\left\{\theta^{a}\right\}_{a \in \mathbb{A}}: \hat{g}^{-0}(\theta) \in \hat{B}\right\}
$$

of all taste shocks $\theta$ such that, in equilibrium, the actions $\hat{g}^{-0}$ of the agents $a \in$ $\{ \pm 2, \pm 1\}$ belong to the set of observed equilibrium actions $\hat{B}$. By analogy to (B.4) and (B.5) independence of the taste shocks yields

$$
\begin{aligned}
\mathbb{P}\left[g^{0} \in \hat{G}(B)\right] & =\int \mathbf{1}_{\hat{H}(B)}\left(\left\{\theta^{a}\right\}_{a \in \mathbb{A}}\right) \prod_{a \in \mathbb{A}} v\left(d \theta^{a}\right) \\
& =\int \mathbb{P}\left[g^{0} \in \hat{G}(B) \mid \theta^{ \pm 1}\right] \prod_{a= \pm 1} v\left(d \theta^{a}\right) .
\end{aligned}
$$

and a representation of the conditional probability of $\left\{g^{0} \in \hat{G}(B)\right\}$, given $\theta^{-1}$ and $\theta^{1}$ :

$$
\begin{aligned}
& \mathbb{P}\left[g^{0} \in \hat{G}(B) \mid \theta^{ \pm 1}\right] \\
& =v\left[\theta^{0}: \text { there exist equilibrium actions } z^{-0} \text { satisfying } \hat{z}^{-0} \in \hat{B}\right. \text { such } \\
& \left.\quad \text { that with } g^{0}=h^{0}\left(\theta^{0}, z^{ \pm 1}\right) \text { we have } z^{ \pm 1}=h^{ \pm 1}\left(\theta^{ \pm 1}, g^{0}, z^{ \pm 2}\right)\right] .
\end{aligned}
$$

(iii) A pair $\left(y^{-0}, z^{-0}\right)$ of equilibrium actions that satisfies $\hat{y}^{-0}=\hat{z}^{-0}$ also satisfies

$$
h^{a}\left(\theta^{a}, y^{a \pm 1}\right)=h^{a}\left(\theta^{a}, z^{a \pm 1}\right) \text { for } a=-1,0,1 \text { and any pair }\left(\theta^{-1}, \theta^{+1}\right) .
$$

This shows that, given the taste shocks $\theta^{-1}$ and $\theta^{-1}$ the following sets are equal:

$\left\{\theta^{0}\right.$ : there exists $y^{-0} \in B$ such that with $g^{0}=h^{0}\left(\theta^{0}, g^{ \pm 1}\right)$ we have

$$
\left.y^{ \pm 1}=h^{ \pm 1}\left(\theta^{ \pm 1}, g^{0}, y^{ \pm 2}\right)\right\}
$$


and

$$
\begin{array}{r}
\left\{\theta^{0}: \text { there exist equilibrium actions } z^{-0} \text { that satisfy } \hat{z}^{-0} \in \hat{B}\right. \text { such } \\
\text { that with } \left.g^{0}=h^{0}\left(\theta^{0}, z^{ \pm 1}\right) \text { we have } z^{ \pm 1}=h^{ \pm 1}\left(\theta^{ \pm 1}, g^{0}, z^{ \pm 2}\right)\right\}
\end{array}
$$

In view of (B.4) and (B.4) this yields

$$
\mathbb{P}\left[g^{0} \in G(B)\right]=\mathbb{P}\left[g^{0} \in \hat{G}(B)\right] .
$$

Similar arguments can now be applied to prove that

$$
\mathbb{P}\left[g^{0} \in A \cap G(B)\right]=\mathbb{P}\left[g^{0} \in A \cap \hat{G}(B)\right],
$$

and so the assertion follows from (B.1) and (B.2).

\section{References}

[1] D. Aliprantis, K.C. Border, Infinite Dimensional Analysis, Springer, Berlin, 1994.

[2] G. Becker, K.M. Murphy, Social Markets: Market Behavior in a Social Environment, Belknap-Harvard University Press, Cambridge, 2001.

[3] A. Bisin, U. Horst, O. Özgür, Rational expectations equilibria of economies with local interactions, J. Econ. Theory, in press.

[4] W.A. Brock, S.N. Durlauf, Discrete choice with social interactions, Rev. Econ. Stud. 68 (2) (2001) 235-260.

[5] W.A. Brock, C. Hommes, A rational route to randomness, Econometrica 65 (5) (1997) 1059-1095.

[6] J. Cooper, A. John, Coordination failures in Keynesian models, Quart. J. Econ. C III (1988) 441-464.

[7] S.N. Durlauf, Non-ergodic economic growth, Rev. Econ. Stud. 60 (1993) 349-366.

[8] S.N. Durlauf, Statistical mechanics approaches to socioeconomic behavior, Reprint No. 455, SSRI, 1997.

[9] G. Ellison, D. Fudemberg, Rules of thumb for social learning, J. Polit. Economy 101 (1993) 612-644.

[10] I.V. Evstigneev, M.I. Taksar, Stochastic equilibria on graphs I, J. Math. Econ. 23 (1994) 401-433.

[11] I.V. Evstigneev, M.I. Taksar, Stochastic equilibria on graphs II, J. Math. Econ. 24 (1995) 383-406.

[12] I.V. Evstigneev, M.I. Taksar, Stochastic economies with locally interacting Agents, Working Paper No. 01-03-018, SSRI, 2001.

[13] H. Föllmer, Random economies with many interacting agents, J. Math. Econ. 1 (1974) 51-62.

[14] H. Föllmer, U. Horst, Convergence of locally and globally interacting Markov chains, Stoch. Proc. Appl. 96 (2001) 99-121.

[15] H. Föllmer, U. Horst, A. Kirman, Equilibria in financial markets with heterogeneous agents: a probabilistic perspective, J. Math. Econ. 41 (1-2) (2004) 123-155.

[16] H.O. Georgii, Gibbs Measures and Phase Transitions, de Gruyter, Berlin, 1988.

[17] E. Glaeser, B. Sacerdote, J. Scheinkman, Crime and social interactions, Quart. J. Econ. C XI (1996) 507-548.

[18] E. Glaeser, J. Scheinkman, Non-market interactions, in: M. Dewatripont, L.P. Hansen, S. Turnovsky (Eds.), Advances in Economics and Econometrics: Theory and Applications, Eight World Congress, Cambridge University Press, Cambridge, 2000.

[19] E. Glaeser, J. Scheinkman, Measuring social interactions, in: S. Durlauf, P. Young (Eds.), Social Dynamics, MIT Press, Cambridge, MA, 2001.

[20] W. Hildenbrand, Random preferences and equilibrium analysis, J. Econ. Theory 3 (1971) 414-429.

[21] U. Horst, Asymptotics of locally interacting Markov chains with global signals, Adv. Appl. Prob. 34 (2) (2002) 1-25.

[22] U. Horst, Financial price fluctuations in a stock market model with many interacting agents, Econ. Theory 25 (4) (2005) 917-932.

[23] U. Horst, J. Scheinkman, A limit theorem for systems of social interactions, in preparation, 2004. 
[24] Y.M. Ioannides, The evolution of trading structures, in: W.B. Arthur, S.M. Durlauf, D. Lane (Eds.), The Economy as an Evolving, Complex System II, SFI Studies in the Sciences of Complexity, Addison-Wesley, Reading, MA, 1997.

[25] Y.M. Ioannides, Topologies of social interactions, Working Paper, Tufts University, 2001.

[26] A. Kirman, C. Oddou, S. Weber, Stochastic communication and coalition formation, Econometrica 54 (1) (1986) 129-138.

[27] T. Lux, The socio-economic dynamics of speculative markets: interacting agents, chaos, and the fat tails of return distributions, J. Econ. Behav. Organ. 33 (1998) 143-165.

[28] E. Malinvaud, The allocation of individual risks in large markets, J. Econ. Theory 4 (1972) 312-328.

[29] S. Morris, Interaction games: a unified analysis of incomplete information, local interaction and random matching, Unpublished, University of Pennsylvania, 1997.

[30] S. Morris, Contagion, Rev. Econ. Stud. 67 (2000) 57-78.

[31] T. Schelling, Dynamic models of segregation, J. Math. Sociology 1 (1971) 143-186.

[32] T. Schelling, A process of residential segregation: neighborhood tipping, in racial discrimination, in: A. Pascal (Ed.), Economic Life, Lexington Books, Lexington, MA, 1972.

[33] K. Yosida, Functional Analysis, Springer, Berlin, 1991. 